\title{
Similarity Solution for Free Convection Flow of a Micropolar Fluid under Convective Boundary Condition via Lie Scaling Group Transformations
}

\author{
Ch. RamReddy, T. Pradeepa, and D. Srinivasacharya \\ Department of Mathematics, National Institute of Technology, Warangal 506004, India \\ Correspondence should be addressed to Ch. RamReddy; chittetiram@gmail.com
}

Received 19 January 2015; Revised 26 April 2015; Accepted 27 April 2015

Academic Editor: Ming Liu

Copyright (C) 2015 Ch. RamReddy et al. This is an open access article distributed under the Creative Commons Attribution License, which permits unrestricted use, distribution, and reproduction in any medium, provided the original work is properly cited. The publication of this article was funded by SCOAP S $^{3}$

\begin{abstract}
The free convective flow of an incompressible micropolar fluid along permeable vertical plate under the convective boundary condition is investigated. The Lie scaling group of transformations is applied to get the similarity representation for the system of partial differential equations and then the resulting systems of equations are solved using spectral quasi-linearisation method. A quantitative comparison of the numerical results is made with previously published results for special cases and the results are found to be in good agreement. The results of the physical parameters on the developments of flow, temperature, concentration, skinfriction, wall couple stress, heat transfer, and mass transfer characteristics along vertical plate are given and the salient features are discussed.
\end{abstract}

\section{Introduction}

In the past few decades, most of the researchers considered convective heat transfer problems with either constant wall temperature (CWT), constant heat flux (CHF), or Newtonian heating $(\mathrm{NH})$ in a Newtonian and/or non-Newtonian fluid. Recently, a novel mechanism for the heating process has drawn the involvement of many researchers, namely, convective boundary condition ( $\mathrm{CBC}$ ), where the heat is supplied to the convecting fluid through a bounding surface with a finite heat capacity. Further, this results in the heat transfer rate through the surface being proportional to the local difference in temperature with the ambient conditions (Merkin [1]). Besides, it is more general and realistic, particularly in various technologies and industrial operations such as transpiration cooling process, textile drying, and laser pulse heating. Aziz [2] reported similarity solution for thermal boundary layer flow over a flat plate in a uniform stream of fluid with the convective boundary condition and he concluded that a similarity solution is possible if the convective heat transfer related to hot fluid on the lower surface of the plate is proportional to the inverse square root of the axial length. In the presence of an internal heat generation local similarity solution for free convection heat transfer from a moving vertical plate with the convective boundary condition is discussed by Makinde [3]. The laminar natural convection flow over a semi-infinite moving vertical plate under the convective boundary condition is examined by Ibrahim and Bhashar Reddy [4]. RamReddy et al. [5] investigated the influence of the prominent Soret effect on mixed convection in a nanofluid under the convective boundary conditions. The nonsimilar result has been presented for the free convection boundary layer flow along a solid sphere under the convective boundary conditions by Alkasasbeh et al. [6]. More recently, a note on the natural convection along convectively heated vertical plate is given by Pantokratoras [7].

One of the best established theories of fluids with microstructure is the theory of micropolar fluids and this theory can be found in the books by Lukaszewicz [8] and Eremeyev et al. [9]. It has gathered a good deal of attention due to the obvious reasons that the Navier Stokes equation for Newtonian fluids cannot successfully explain the attributes of 
fluids with a substructure. Physically, the micropolar fluids may be treated as non-Newtonian fluids consisting of dumbbell molecules or rigid cylindrical element, polymer fluids, fluid suspension, animal blood, and so forth. Further, the theory of micropolar fluids includes microrotation as well as microinertia effects. This theory studies viscous fluids in which microconstituents are rigid and spherical or randomly oriented as well. The subject of free convection boundary layer flow in a micropolar fluid has been keyed out by several investigators due to its immense applications in the engineering problems such as solar energy collecting devices, air conditioning of a room, material processing, and passive cooling of nuclear reactors. The boundary layer flow over a semi-infinite flat plate is considered for analyzing theory of micropolar fluid and its application to low concentration suspension flow by Ahmadi [10]. Rees and Pop [11] discussed the free convection boundary layer flow of a micropolar fluid from a vertical flat plate. The nonsimilarity transformations are used to analyze the effects of double stratification on free/mixed convective transport in a micropolar fluid by Srinivasacharya and RamReddy [12-14] (also see the references cited therein). The problems of a steady laminar stagnation point flow towards a stretching/shrinking sheet in an incompressible micropolar fluid under the convective surface boundary condition are discussed by Yacob and Ishak [15] and Zaimi and Ishak [16]. Merely from the literature, it is noted that the majority of the researchers have found the local similarity or nonsimilarity solutions for the problems involving convective boundary conditions, since most of the researchers have taken a convective heat transfer coefficient as a function $x$ for getting the similarity solutions in their problems. Nevertheless, the assumption of a heat transfer coefficient varying along the plate as a function of $x$ is not realistic and very difficult to be obtained in practice. For that cause, it could be supposed that the above works have only theoretical value.

In the recent past, several researchers are focused on obtaining the similarity solutions of the convective transport phenomena problems arising in fluid dynamics, aerodynamics, plasma physics, meteorology, and some branches of engineering by using different procedures. One such procedure is Lie group analysis. The concept of Lie group analysis also called symmetry analysis is developed by Sophius Lie to determine transformations which map a given differential equation to itself and it unifies almost all known exact integration techniques (see [17-19]). It provides a potent, sophisticated, and systematic tool for generating the invariant solutions of the system of nonlinear partial differential equations (PDEs) with relevant initial or boundary conditions. A special form of Lie group transformations, known as the scaling group, has been suggested by various researchers to study convection flows of different flow phenomena (see Tapanidis et al. [20], Hassanien and Hamad [21], Kandasamy et al. [22], Aziz et al. [23], Mutlag et al. [24], etc.; they are worth observing).

From the literature survey, it seems that the problem of the free convective heat and mass transport along permeable vertical plate in a micropolar fluid under the convective boundary condition has not been investigated so far. Motivated by all these works, this paper attempts to present the new similarity transformations and corresponding similarity solution to investigate the free convection flow of a micropolar fluid under the convective boundary condition using the Lie group transformations. The mathematical model involving the convective boundary conditions becomes slightly more complicated leading to the complex interactions of the flow, heat, and mass transfer mechanism. Further, the analytical solution is out of scope in the present set-up and hence a numerical solution is obtained for the current problem. Also, the influence of important parameters, namely, micropolar, suction/injection, and convective heat transfer parameters, on the physical quantities of the flow, heat, and mass transfer rates is analyzed in different flow situations.

\section{Mathematical Formulation}

Consider the steady, laminar, and free convective flow of an incompressible micropolar fluid with the free stream temperature and concentration, $T_{\infty}$ and $C_{\infty}$, respectively. Choose the coordinate system such that the $\bar{x}$-axis is along the vertical plate and $\bar{y}$-axis normal to the plate, as shown in Figure 1. The suction/injection velocity distribution is assumed to be $v_{w}$. The plate is either heated or cooled from left by convection from a fluid of temperature $T_{f}$ with $T_{f}>$ $T_{\infty}$ corresponding to a heated surface (assisting flow) and $T_{f}<T_{\infty}$ corresponding to a cooled surface (opposing flow), respectively. On the wall concentration is taken to be constant and is given by $C_{w}$.

By employing Boussinesq approximation and making use of the standard boundary layer approximations, the governing equations for the micropolar fluid [10] are given by

$$
\begin{aligned}
& \frac{\partial \bar{u}}{\partial \bar{x}}+\frac{\partial \bar{v}}{\partial \bar{y}}=0 \\
& \rho\left(\bar{u} \frac{\partial \bar{u}}{\partial \bar{x}}+\bar{v} \frac{\partial \bar{u}}{\partial \bar{y}}\right) \\
& =(\mu+\kappa) \frac{\partial^{2} \bar{u}}{\partial \bar{y}^{2}}+\kappa \frac{\partial \bar{\omega}}{\partial \bar{y}} \\
& \quad+\rho g^{*}\left(\beta_{T}(\bar{x})\left(T-T_{\infty}\right)+\beta_{C}(\bar{x})\left(C-C_{\infty}\right)\right), \\
& \rho j\left(\bar{u} \frac{\partial \bar{\omega}}{\partial \bar{x}}+\bar{v} \frac{\partial \bar{\omega}}{\partial \bar{y}}\right)=\gamma \frac{\partial^{2} \bar{\omega}}{\partial \bar{y}^{2}}-\kappa\left(2 \bar{\omega}+\frac{\partial \bar{u}}{\partial \bar{y}}\right), \\
& \bar{u} \frac{\partial T}{\partial \bar{x}}+\bar{v} \frac{\partial T}{\partial \bar{y}}=\alpha \frac{\partial^{2} T}{\partial \bar{y}^{2}}, \\
& \bar{u} \frac{\partial C}{\partial \bar{x}}+\bar{v} \frac{\partial C}{\partial \bar{y}}=D \frac{\partial^{2} C}{\partial \bar{y}^{2}},
\end{aligned}
$$

where $\bar{u}$ and $\bar{v}$ are the velocity components in $\bar{x}$ and $\bar{y}$ directions, respectively, $\bar{\omega}$ is the component of microrotation whose direction of rotation lies in the $\bar{x} \bar{y}$-plane, $T$ is the temperature, $C$ is the concentration, $g^{*}$ is the acceleration due to gravity, $\rho$ is the density, $\mu$ is the dynamic coefficient 


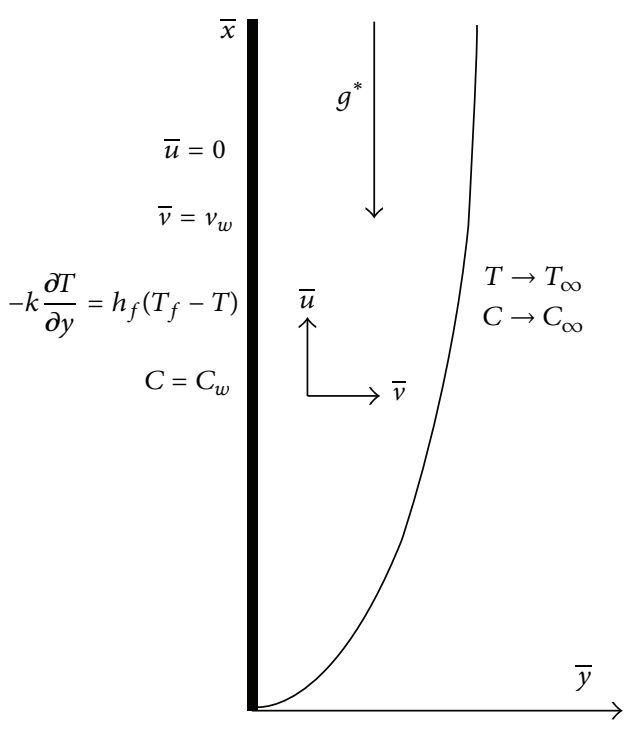

FIGURE 1: Physical model and coordinate system.

of viscosity, $\beta_{T}(\bar{x})$ is the volumetric coefficient of thermal expansion, $\beta_{C}(\bar{x})$ is the volumetric coefficient of solutal expansions, $\kappa$ is the vortex viscosity, $j$ is the microinertia density, $\gamma$ is the spin-gradient viscosity, $\alpha$ is the thermal diffusivity, and $D$ is the solutal diffusivity of the medium.

The boundary conditions are

$$
\begin{aligned}
\bar{u} & =0, \\
\bar{v} & =v_{w}, \\
\bar{\omega} & =-n \frac{\partial \bar{u}}{\partial \bar{y}}, \\
-k \frac{\partial T}{\partial \bar{y}} & =h_{f}\left(T_{f}-T\right), \\
C & =C_{w}, \\
\text { at } \bar{y} & =0, \\
\bar{u} & =0, \\
\bar{\omega} & =0, \\
T & =T_{\infty}, \\
C & =C_{\infty}, \\
\text { as } \bar{y} & \longrightarrow \infty,
\end{aligned}
$$

where subscripts $w$ and $\infty$ indicate the conditions at the wall and at the outer edge of the boundary layer, respectively, $h_{f}$ is the convective heat transfer coefficient, $k$ is the thermal conductivity of the fluid, and $n$ is a material constant. Further, we follow the work of many recent authors by assuming that $\gamma=(\mu+\kappa / 2) j$. This assumption is invoked to allow the field of equations to predict the correct behavior in the limiting case when the microstructure effects become negligible and the total spin $\bar{\omega}$ reduces to the angular velocity [10].

\section{Nondimensionalization of the Governing Equations}

Introduce the following dimensionless variables:

$$
\begin{aligned}
& x=\frac{\bar{x}}{L}, \\
& y=\frac{\bar{y}}{L} \mathrm{Gr}^{1 / 4}, \\
& u=\frac{L}{\nu \mathrm{Gr}^{1 / 2}} \bar{u}, \\
& v=\frac{L}{\nu \mathrm{Gr}^{1 / 4}} \bar{v}, \\
& \omega=\frac{L^{2}}{\nu \mathrm{Gr}^{3 / 4}} \bar{\omega}, \\
& \theta=\frac{T-T_{\infty}}{T_{f}-T_{\infty}}, \\
& \phi=\frac{C-C_{\infty}}{C_{w}-C_{\infty}},
\end{aligned}
$$

where $\mathrm{Gr}=g^{*} \beta_{T_{0}}\left(T_{f}-T_{\infty}\right) L^{3} / \nu^{2}$ is the Grashof number.

In view of the continuity equation (1), we introduce the stream function $\psi$ by

$$
\begin{aligned}
& u=\frac{\partial \psi}{\partial y} \\
& v=-\frac{\partial \psi}{\partial x}
\end{aligned}
$$

Using (7) and (8) into (2)-(5), we get the following momentum, angular momentum, energy, and concentration equations:

$$
\begin{aligned}
\Delta_{1}= & \frac{\partial \psi}{\partial y} \frac{\partial^{2} \psi}{\partial x \partial y}-\frac{\partial \psi}{\partial x} \frac{\partial^{2} \psi}{\partial y^{2}}-\left(\frac{1}{1-N}\right)\left[\frac{\partial^{3} \psi}{\partial y^{3}}-N \frac{\partial \omega}{\partial y}\right] \\
& -\frac{g^{*} \beta_{T}(x)\left(T_{f}-T_{\infty}\right) L^{3}}{\nu^{2} \mathrm{Gr}} \theta \\
& -\frac{g^{*} \beta_{C}(x)\left(C_{w}-C_{\infty}\right) L^{3}}{\nu^{2} \mathrm{Gr}} \phi=0, \\
\Delta_{2}= & \frac{\partial \psi}{\partial y} \frac{\partial \omega}{\partial x}-\frac{\partial \psi}{\partial x} \frac{\partial \omega}{\partial y}-\left(\frac{2-N}{2-2 N}\right) \frac{\partial^{2} \omega}{\partial y^{2}} \\
& +\left(\frac{N}{1-N}\right)\left[2 \omega+\frac{\partial^{2} \psi}{\partial y^{2}}\right]=0, \\
\Delta_{3}= & \frac{\partial \psi}{\partial y} \frac{\partial \theta}{\partial x}-\frac{\partial \psi}{\partial x} \frac{\partial \theta}{\partial y}-\frac{1}{\operatorname{Pr}} \frac{\partial^{2} \theta}{\partial y^{2}}=0, \\
\Delta_{4}= & \frac{\partial \psi}{\partial y} \frac{\partial \phi}{\partial x}-\frac{\partial \psi}{\partial x} \frac{\partial \phi}{\partial y}-\frac{1}{\operatorname{Sc}} \frac{\partial^{2} \phi}{\partial y^{2}}=0 .
\end{aligned}
$$

In usual definitions, $v$ is the kinematic viscosity, $\operatorname{Pr}=v / \alpha$ is the Prandtl number, $\mathrm{Sc}=\nu / D$ is the Schmidt number, $N=$ $\kappa /(\mu+\kappa)(0 \leq N<1)$ is the coupling number [25], and the microinertia density is taken to be $j=L^{2} / \mathrm{Gr}^{1 / 2}$. 
Now boundary conditions (6a) and (6b) become

$$
\begin{aligned}
\frac{\partial \psi}{\partial y} & =0 \\
\frac{\partial \psi}{\partial x} & =f_{w} \\
\omega & =-n \frac{\partial^{2} \psi}{\partial y^{2}}, \\
\frac{\partial \theta}{\partial y} & =-\operatorname{Bi}(1-\theta), \\
\phi & =1 \\
\text { at } y & =0 \\
\frac{\partial \psi}{\partial y} & =0 \\
\omega & =0 \\
\theta & =0 \\
\phi & =0 \\
\text { as } y & \longrightarrow \infty
\end{aligned}
$$

where $f_{w}=-\left(L / \nu \mathrm{Gr}^{1 / 4}\right) v_{w}$ is the suction/injection parameter. It is worth mentioning that $f_{w}$ determines the transpiration rate at the surface, with $f_{w}>0$ for suction and $f_{w}<0$ for injection, and $f_{w}=0$ corresponds to an impermeable surface. Further, $\mathrm{Bi}=h_{f} L / k \mathrm{Gr}^{1 / 4}$ is the Biot number. It is a ratio of the internal thermal resistance of the plate to the boundary layer thermal resistance of the hot fluid at the bottom of the surface.

\section{Similarity Equations via Lie Scaling Group Transformations}

A one-parameter Lie scaling group of transformations, which is a simplified form of Lie group transformation, is selected as (for more, see [26-31])

$$
\begin{aligned}
\Gamma: x^{*} & =x e^{\varepsilon \alpha_{1}}, \\
y^{*} & =y e^{\varepsilon \alpha_{2}}, \\
\psi^{*} & =\psi e^{\varepsilon \alpha_{3}}, \\
\omega^{*} & =\omega e^{\varepsilon \alpha_{4}}, \\
\theta^{*} & =\theta e^{\varepsilon \alpha_{5}}, \\
\phi^{*} & =\phi e^{\varepsilon \alpha_{6}}, \\
\beta_{T}^{*} & =\beta_{T} e^{\varepsilon \alpha_{7}}, \\
\beta_{C}^{*} & =\beta_{C} e^{\varepsilon \alpha_{8}} .
\end{aligned}
$$

Here $\varepsilon \neq 0$ is the parameter of the group and $\alpha_{i}$ (where $i=1,2,3, \ldots, 8)$ are arbitrary real numbers whose interrelationship will be determined by our analysis. Transformations in (11) may be treated as a point transformation, transforming the coordinates

$$
\begin{aligned}
& \left(x, y, \psi, \omega, \theta, \phi, \beta_{T}, \beta_{C}\right) \\
& \quad=\left(x^{*}, y^{*}, \psi^{*}, \omega^{*}, \theta^{*}, \phi^{*}, \beta_{T}^{*}, \beta_{C}^{*}\right) .
\end{aligned}
$$

We now investigate the relationship among the exponents $\alpha_{i}$ (where $i=1,2,3, \ldots, 8$ ) such that

$$
\begin{aligned}
& \Delta_{j}\left[x^{*}, y^{*}, u^{*}, v^{*}, \ldots, \frac{\partial^{3} \psi^{*}}{\partial y^{* 3}}\right] \\
& \quad=H_{j}\left[x, y, u, v, \ldots, \frac{\partial^{3} \psi}{\partial y^{3}} ; a\right] \\
& \cdot \Delta_{j}\left[x, y, u, v, \ldots, \frac{\partial^{3} \psi}{\partial y^{3}}\right], \quad(j=1,2,3,4) .
\end{aligned}
$$

This is the requirement that the differential forms $\Delta_{1}, \Delta_{2}, \Delta_{3}$, and $\Delta_{4}$ are conformally invariant under transformation (11). Substituting transformations (11) in (9), we have

$$
\begin{aligned}
& \Delta_{1}=e^{\varepsilon\left(\alpha_{1}+2 \alpha_{2}-2 \alpha_{3}\right)}\left(\frac{\partial \psi^{*}}{\partial y^{*}} \frac{\partial^{2} \psi^{*}}{\partial x^{*} \partial y^{*}}-\frac{\partial \psi^{*}}{\partial x^{*}} \frac{\partial^{2} \psi^{*}}{\partial y^{* 2}}\right) \\
& -\left(\frac{1}{1-N}\right) e^{\varepsilon\left(3 \alpha_{2}-\alpha_{3}\right)} \frac{\partial^{3} \psi^{*}}{\partial y^{* 3}} \\
& -\left(\frac{N}{1-N}\right) e^{\varepsilon\left(\alpha_{2}-\alpha_{4}\right)} \frac{\partial \omega^{*}}{\partial y^{*}} \\
& -\frac{g^{*} \beta_{T}^{*}\left(T_{f}-T_{\infty}\right) L^{3}}{v^{2} \mathrm{Gr}} e^{-\varepsilon\left(\alpha_{5}+\alpha_{7}\right)} \theta^{*} \\
& -\frac{g^{*} \beta_{C}^{*}\left(C_{w}-C_{\infty}\right) L^{3}}{v^{2} \mathrm{Gr}} e^{-\varepsilon\left(\alpha_{6}+\alpha_{8}\right)} \phi^{*}=0, \\
& \Delta_{2}=e^{\varepsilon\left(\alpha_{1}+\alpha_{2}-\alpha_{3}-\alpha_{4}\right)}\left(\frac{\partial \psi^{*}}{\partial y^{*}} \frac{\partial \omega^{*}}{\partial x^{*}}-\frac{\partial \psi^{*}}{\partial x^{*}} \frac{\partial \omega^{*}}{\partial y^{*}}\right) \\
& -\left(\frac{2-N}{2-2 N}\right) e^{\varepsilon\left(2 \alpha_{2}-\alpha_{4}\right)} \frac{\partial^{2} \omega^{*}}{\partial y^{* 2}} \\
& +\left(\frac{N}{1-N}\right)\left(2 \omega^{*} e^{-\varepsilon \alpha_{4}}+e^{\varepsilon\left(2 \alpha_{2}-\alpha_{3}\right)} \frac{\partial^{2} \psi^{*}}{\partial y^{* 2}}\right) \\
& =0 \text {, } \\
& \Delta_{3}=e^{\varepsilon\left(\alpha_{1}+\alpha_{2}-\alpha_{3}-\alpha_{5}\right)}\left(\frac{\partial \psi^{*}}{\partial y^{*}} \frac{\partial \theta^{*}}{\partial x^{*}}-\frac{\partial \psi^{*}}{\partial x^{*}} \frac{\partial \theta^{*}}{\partial y^{*}}\right) \\
& -\frac{1}{\operatorname{Pr}} e^{\varepsilon\left(2 \alpha_{2}-\alpha_{5}\right)}\left(\frac{\partial^{2} \theta^{*}}{\partial y^{* 2}}\right)=0, \\
& \Delta_{4}=e^{\varepsilon\left(\alpha_{1}+\alpha_{2}-\alpha_{3}-\alpha_{6}\right)}\left(\frac{\partial \psi^{*}}{\partial y^{*}} \frac{\partial \phi^{*}}{\partial x^{*}}-\frac{\partial \psi^{*}}{\partial x^{*}} \frac{\partial \phi^{*}}{\partial y^{*}}\right) \\
& -\frac{1}{\mathrm{Sc}} e^{\varepsilon\left(2 \alpha_{2}-\alpha_{6}\right)}\left(\frac{\partial^{2} \phi^{*}}{\partial y^{* 2}}\right)=0 .
\end{aligned}
$$


Now, boundary conditions (10a) and (10b) become

$$
\begin{aligned}
e^{\varepsilon\left(\alpha_{2}-\alpha_{3}\right)} \frac{\partial \psi^{*}}{\partial y^{*}} & =0, \\
e^{\varepsilon\left(\alpha_{1}-\alpha_{3}\right)} \frac{\partial \psi^{*}}{\partial x^{*}} & =f_{w}, \\
e^{-\varepsilon \alpha_{4}} \omega^{*} & =-n e^{\varepsilon\left(2 \alpha_{2}-\alpha_{3}\right)} \frac{\partial^{2} \psi^{*}}{\partial y^{* 2}}, \\
e^{\varepsilon\left(\alpha_{2}-\alpha_{5}\right)} \frac{\partial \theta^{*}}{\partial y^{*}} & =-\operatorname{Bi}\left(1-e^{-\varepsilon \alpha_{5}} \theta^{*}\right), \\
e^{-\varepsilon \alpha_{6}} \phi^{*} & =1, \\
\text { at } y^{*} & =0, \\
e^{\varepsilon\left(\alpha_{2}-\alpha_{3}\right)} \frac{\partial \psi^{*}}{\partial y^{*}} & =0, \\
e^{-\varepsilon \alpha_{4}} \omega^{*} & =0, \\
e^{-\varepsilon \alpha_{5}} \theta^{*} & =0, \\
e^{-\varepsilon \alpha_{6}} \phi^{*} & =0, \\
\text { as } y^{*} & \longrightarrow \infty .
\end{aligned}
$$

The system remains invariant under the group transformation $\Gamma$. We then have the following relationships for the parameters:

$$
\begin{aligned}
\alpha_{1}+2 \alpha_{2}-2 \alpha_{3} & =3 \alpha_{2}-\alpha_{3}=\alpha_{2}-\alpha_{4}=-\alpha_{5}-\alpha_{7} \\
& =-\alpha_{6}-\alpha_{8} \\
\alpha_{1}+\alpha_{2}-\alpha_{3}-\alpha_{4} & =2 \alpha_{2}-\alpha_{4}=-\alpha_{4}=2 \alpha_{2}-\alpha_{3} \\
\alpha_{1}+\alpha_{2}-\alpha_{3}-\alpha_{5} & =2 \alpha_{2}-\alpha_{5} \\
\alpha_{1}+\alpha_{2}-\alpha_{3}-\alpha_{6} & =2 \alpha_{2}-\alpha_{6} \\
\alpha_{1}-\alpha_{3} & =0 \\
-\alpha_{4} & =2 \alpha_{2}-\alpha_{3} \\
\alpha_{2}-\alpha_{5} & =0=-\alpha_{5} \\
\alpha_{6} & =0
\end{aligned}
$$

Solving linear system (16), we have the following relationship among the exponents:

$$
\begin{aligned}
& \alpha_{1}=\alpha_{3}=\alpha_{4}=\alpha_{7}=\alpha_{8}, \\
& \alpha_{2}=\alpha_{5}=\alpha_{6}=0 .
\end{aligned}
$$

The set of transformations $\Gamma$ reduces to

$$
\begin{aligned}
& x^{*}=x e^{\varepsilon \alpha_{1},} \\
& y^{*}=y, \\
& \psi^{*}=\psi e^{\varepsilon \alpha_{1}}, \\
& \omega^{*}=\omega e^{\varepsilon \alpha_{1},} \\
& \theta^{*}=\theta, \\
& \phi^{*}=\phi, \\
& \beta_{T}^{*}=\beta_{T} e^{\varepsilon \alpha_{1}}, \\
& \beta_{C}^{*}=\beta_{C} e^{\varepsilon \alpha_{1}} .
\end{aligned}
$$

Expanding by the Taylor series in power of $\varepsilon$, keeping the term up to the first degree (neglecting higher power of $\varepsilon$ ), we get

$$
\begin{aligned}
x^{*}-x & =\varepsilon \alpha_{1} x, \\
y^{*} & =y, \\
\psi^{*}-\psi & =\varepsilon \alpha_{1} \psi, \\
\omega^{*}-\omega & =\varepsilon \alpha_{1} \omega, \\
\theta^{*} & =\theta, \\
\phi^{*} & =\phi, \\
\beta_{T}^{*}-\beta_{T} & =\varepsilon \alpha_{1} \beta_{T}, \\
\beta_{C}^{*}-\beta_{C} & =\varepsilon \alpha_{1} \beta_{C} .
\end{aligned}
$$

The characteristic equations are

$$
\begin{aligned}
\frac{d x}{\alpha_{1} x} & =\frac{d y}{0}=\frac{d \psi}{\alpha_{1} \psi}=\frac{d \omega}{\alpha_{1} \omega}=\frac{d \theta}{0}=\frac{d \phi}{0}=\frac{d \beta_{T}}{\alpha_{1} \beta_{T}} \\
& =\frac{d \beta_{C}}{\alpha_{1} \beta_{C}} .
\end{aligned}
$$

Solving the above characteristic equations, we have the following similarity transformations:

$$
\begin{aligned}
\eta & =y, \\
\psi & =x f(\eta), \\
\omega & =x g(\eta), \\
\beta_{T} & =\beta_{T_{0}} x, \\
\beta_{C} & =\beta_{C_{0}} x, \\
\theta & =\theta(\eta), \\
\phi & =\phi(\eta),
\end{aligned}
$$

where $\beta_{T_{0}}$ and $\beta_{C_{0}}$ are constant thermal and mass coefficient of expansion.

Using (21) into (9), we get the following similarity equations:

$$
\begin{aligned}
& \left(\frac{1}{1-N}\right) f^{\prime \prime \prime}+f f^{\prime \prime}-f^{\prime 2}+\left(\frac{N}{1-N}\right) g^{\prime}+\theta+\mathscr{B} \phi \\
& \quad=0 \\
& \left(\frac{2-N}{2-2 N}\right) g^{\prime \prime}+f g^{\prime}-f^{\prime} g-\left(\frac{N}{1-N}\right)\left(2 g+f^{\prime \prime}\right) \\
& \quad=0, \\
& \frac{1}{\operatorname{Pr}} \theta^{\prime \prime}+f \theta^{\prime}=0, \\
& \frac{1}{\mathrm{Sc}} \phi^{\prime \prime}+f \phi^{\prime}=0,
\end{aligned}
$$

where the primes indicate differentiation with respect to $\eta$ alone and $\mathscr{B}=\beta_{C_{0}}\left(C_{w}-C_{\infty}\right) / \beta_{T_{0}}\left(T_{f}-T_{\infty}\right)$ is the buoyancy ratio. 
Boundary conditions (10a) and (10b) in terms of $f, g, \theta$, and $\phi$ become

$$
\begin{aligned}
\eta & =0: f(0)=f_{w}, \\
f^{\prime}(0) & =0, \\
g(0) & =-n f^{\prime \prime}(0), \\
\theta^{\prime}(0) & =-\operatorname{Bi}[1-\theta(0)], \\
\phi(0) & =1, \\
\eta & \longrightarrow \infty: f^{\prime}(\infty)=0, \\
g(\infty) & =0, \\
\theta(\infty) & =0, \\
\phi(\infty) & =0 .
\end{aligned}
$$

\section{Skin Friction, Wall Couple Stress, and Heat and Mass Transfer Coefficients}

The wall shear stress and the wall couple stress are

$$
\begin{aligned}
\tau_{w} & =\left[(\mu+\kappa) \frac{\partial \bar{u}}{\partial \bar{y}}+\kappa \bar{\omega}\right]_{\bar{y}=0}, \\
m_{w} & =\gamma\left[\frac{\partial \bar{\omega}}{\partial \bar{y}}\right]_{\bar{y}=0},
\end{aligned}
$$

and the heat and mass transfers from the plate, respectively, are given by

$$
\begin{aligned}
& q_{w}=-k\left[\frac{\partial T}{\partial \bar{y}}\right]_{\bar{y}=0}, \\
& q_{m}=-D\left[\frac{\partial C}{\partial \bar{y}}\right]_{\bar{y}=0} .
\end{aligned}
$$

The nondimensional skin friction $C_{f}=2 \tau_{w} / \rho \bar{u}_{*}^{2}$, wall couple stress $M_{w}=m_{w} / \rho \bar{u}_{*}^{2} \bar{x}$, the local Nusselt number $N u_{\bar{x}}=q_{w} \bar{x} / k\left(T_{f}-T_{\infty}\right)$, and local Sherwood number $S h_{\bar{x}}=$ $q_{m} \bar{x} / D\left(C_{w}-C_{\infty}\right)$ are given by

$$
\begin{aligned}
C_{f} \mathrm{Gr}_{\bar{x}}^{1 / 4} & =2\left(\frac{1-n N}{1-N}\right) f^{\prime \prime}(0), \\
M_{w} \mathrm{Gr}_{\bar{x}}^{1 / 2} & =\left(\frac{2-N}{2-2 N}\right) g^{\prime}(0), \\
\frac{N u_{\bar{x}}}{\mathrm{Gr}_{\bar{x}}^{1 / 4}} & =-\theta^{\prime}(0), \\
\frac{\mathrm{Sh}_{\bar{x}}}{\mathrm{Gr}_{\bar{x}}^{1 / 4}} & =-\phi^{\prime}(0),
\end{aligned}
$$

where $\bar{u}_{*}^{2}$ is the characteristic velocity and $\mathrm{Gr}_{\bar{x}}=g^{*} \beta_{T_{0}}\left(T_{f}-\right.$ $\left.T_{\infty}\right) \bar{x}^{3} / \nu^{2}$ is the local Grashof number.

\section{Numerical Solution Using the Spectral Quasi-Linearization Method (SQLM)}

In this section, we describe the quasi-linearization method (QLM) for solving the governing system of (22) along with boundary conditions (23a) and (23b). This QLM is a generalization of the Newton-Raphson method and was proposed by Bellman and Kalaba [32] for solving nonlinear boundary value problems.

Assume that the solutions $f_{r}, g_{r}, \theta_{r}$, and $\phi_{r}$ of (22) at the $(r+1)$ th iteration are $f_{r+1}, g_{r+1}, \theta_{r+1}$, and $\phi_{r+1}$. If the solutions at the previous iteration are sufficiently close to the solutions at the present iteration, the nonlinear components of (22) can be linearised using one-term Taylor series of multiple variables so that (22) give the following iterative sequence of linear differential equations:

$$
\begin{aligned}
& \left(\frac{1}{1-N}\right) f_{r+1}^{\prime \prime \prime}+a_{1, r} f_{r+1}^{\prime \prime}+a_{2, r} f_{r+1}^{\prime}+a_{3, r} f_{r+1} \\
& +\left(\frac{N}{1-N}\right) g_{r+1}^{\prime}+\theta_{r+1}+\mathscr{B} \phi_{r+1}=R_{1, r}, \\
& \left(\frac{2-N}{2-2 N}\right) g_{r+1}^{\prime \prime}+b_{3, r} g_{r+1}^{\prime}+b_{4, r} g_{r+1}+b_{1, r} f_{r+1}^{\prime} \\
& +b_{2, r} f_{r+1}-\left(\frac{N}{1-N}\right) f_{r+1}^{\prime \prime}=R_{2, r}, \\
& c_{1, r} f_{r+1}+\frac{1}{\operatorname{Pr}} \theta_{r+1}^{\prime \prime}+c_{2, r} \theta_{r+1}^{\prime}=R_{3, r}, \\
& d_{1, r} f_{r+1}+\frac{1}{\mathrm{Sc}} \phi_{r+1}^{\prime \prime}+d_{2, r} \phi_{r+1}^{\prime}=R_{4, r},
\end{aligned}
$$

where the coefficients $a_{s_{1}, r}\left(s_{1}=1,2,3\right), b_{s_{2}, r}\left(s_{2}=1,2, \ldots\right.$, 4), $c_{s_{3}, r}\left(s_{3}=1,2\right), d_{s_{4}, r}\left(s_{4}=1,2\right)$, and $R_{s_{5}, r}\left(s_{5}=1,2, \ldots\right.$, 4) are known functions (from previous iterations) and are defined as

$$
\begin{aligned}
a_{1, r} & =f_{r}, \\
a_{2, r} & =-2 f_{r}^{\prime}, \\
a_{3, r} & =f_{r}^{\prime \prime}, \\
R_{1, r} & =f_{r} f_{r}^{\prime \prime}-\left(f_{r}^{\prime}\right)^{2}, \\
b_{1, r} & =-g_{r}, \\
b_{2, r} & =g_{r}^{\prime}, \\
b_{3, r} & =f_{r}, \\
b_{4, r} & =-f_{r}^{\prime}-\left(\frac{2 N}{1-N}\right), \\
R_{2, r} & =f_{r} g_{r}^{\prime}-f_{r}^{\prime} g_{r}, \\
c_{1, r} & =\theta_{r}^{\prime}, \\
c_{2, r} & =f_{r}, \\
R_{3, r} & =f_{r} \theta_{r}^{\prime}, \\
d_{1, r} & =\phi_{r}^{\prime}, \\
d_{2, r} & =f_{r}, \\
R_{4, r} & =f_{r} \phi_{r}^{\prime},
\end{aligned}
$$


subject to boundary conditions

$$
\begin{aligned}
f_{r+1}(0) & =f_{w}, \\
f_{r+1}^{\prime} & =0, \\
f_{r+1}^{\prime}(\infty) & =0, \\
g_{r+1} & =-n f_{r+1}^{\prime \prime}(0), \\
g_{r+1}(\infty) & =0, \\
\theta_{r+1}^{\prime}(0) & =-\operatorname{Bi}(1-\theta(0)), \\
\theta_{r+1}(\infty) & =0, \\
\phi_{r+1}(0) & =1, \\
\phi_{r+1}(\infty) & =0 .
\end{aligned}
$$

System (26) constitutes a linear system of coupled differential equations with variable coefficients and can be solved iteratively using any numerical method for $r=1,2,3, \ldots$. In this work, as will be discussed below, the Chebyshev pseudospectral method was used to solve the QLM scheme (26) (for more details, refer to the works of Motsa et al. $[33,34])$ :

$$
\begin{aligned}
f_{0}(\eta) & =f_{w}+1-e^{-\eta}, \\
g_{0}(\eta) & =-n e^{-\eta} \\
\theta_{0} & =e^{-\eta} \frac{\mathrm{Bi}}{\mathrm{Bi}+1}, \\
\phi_{0} & =e^{-\eta}
\end{aligned}
$$

and starting from these sets of initial approximations $f_{0}, g_{0}$, $\theta_{0}$, and $\phi_{0}$, the iteration schemes (26) can be solved iteratively for $f_{r+1}(\eta), g_{r+1}(\eta), \theta_{r+1}(\eta)$, and $\phi_{r+1}(\eta)$ when $r=0,1,2, \ldots$. For this, we discretise the equation using the Chebyshev spectral collocation method. The unknown functions are approximated by the Chebyshev interpolating polynomials in such way that they are collocated at the Gauss-Lobatto collocation points defined as

$$
\tau_{j}=\cos \frac{\pi j}{\bar{N}}, \quad j=0,1,2, \ldots, \bar{N}
$$

where $\bar{N}$ is the number of collocation points. The physical region $[0, \infty)$ is transformed into the region $[-1,1]$ using the domain truncation technique in which the problem is solved on the interval $\left[0, \eta_{\infty}\right]$ instead of $[0, \infty)$. This leads to the mapping

$$
\frac{\eta}{\eta_{\infty}}=\frac{\tau+1}{2}, \quad-1 \leq \tau \leq 1,
$$

where $\eta_{\infty}$ is the scaling parameter used to invoke the boundary condition at infinity. The functions $f, g, \theta$, and $\phi$ are approximated at the collocation points by

$$
\begin{aligned}
& f(\tau)=\sum_{k=0}^{\bar{N}} f\left(\tau_{k}\right) T_{k}\left(\tau_{j}\right), \\
& g(\tau)=\sum_{k=0}^{\bar{N}} g\left(\tau_{k}\right) T_{k}\left(\tau_{j}\right), \\
& \theta(\tau)=\sum_{k=0}^{\bar{N}} \theta\left(\tau_{k}\right) T_{k}\left(\tau_{j}\right), \\
& \phi(\tau)=\sum_{k=0}^{\bar{N}} \phi\left(\tau_{k}\right) T_{k}\left(\tau_{j}\right), \\
& j=0,1,2, \ldots, \bar{N},
\end{aligned}
$$

where $T_{k}$ is the $k$ th Chebyshev polynomial defined as

$$
T_{k}(\tau)=\cos \left[k \cos ^{-1}(\tau)\right] .
$$

The derivatives of the variables at the collocation points are represented as

$$
\begin{array}{r}
\frac{d^{p} f}{d \eta^{p}}=\sum_{k=0}^{\bar{N}} \mathbf{D}_{l k}^{p} f\left(\tau_{k}\right), \\
\frac{d^{p} g}{d \eta^{p}}=\sum_{k=0}^{\bar{N}} \mathbf{D}_{l k}^{p} g\left(\tau_{k}\right), \\
\frac{d^{p} \theta}{d \eta^{p}}=\sum_{k=0}^{\bar{N}} \mathbf{D}_{l k}^{p} \theta\left(\tau_{k}\right), \\
\frac{d^{p} \phi}{d \eta^{p}}=\sum_{k=0}^{\bar{N}} \mathbf{D}_{l k}^{p} \phi\left(\tau_{k}\right), \\
l=0,1, \ldots, \bar{N},
\end{array}
$$

where $p$ is the order of the derivative and $\mathbf{D}=2 \mathscr{D} / \eta_{\infty}$ is the Chebyshev spectral differentiation matrix and its entries are clearly defined in Canuto et al. [35].

Substituting (31)-(34) into (26) leads to the matrix equation

$$
A X=R,
$$

subject to the boundary conditions

$$
\begin{aligned}
f_{r+1}\left(\tau_{\bar{N}}\right) & =f_{w}, \\
\sum_{k=0}^{\bar{N}} \mathbf{D}_{\bar{N} k} f\left(\tau_{k}\right) & =0, \\
\sum_{k=0}^{\bar{N}} \mathbf{D}_{0 k} f\left(\tau_{k}\right) & =0, \\
g_{r+1}\left(\tau_{\bar{N}}\right) & =-n \sum_{k=0}^{\bar{N}} \mathbf{D}_{\bar{N} k}^{2} f\left(\tau_{k}\right), \\
g_{r+1}\left(\tau_{0}\right) & =0,
\end{aligned}
$$




$$
\begin{aligned}
\sum_{k=0}^{\bar{N}} \mathbf{D}_{\bar{N} k} \theta_{r+1}\left(\tau_{k}\right)-\operatorname{Bi} \theta_{r+1}\left(\tau_{\bar{N}}\right) & =-\mathrm{Bi}, \\
\theta_{r+1}\left(\tau_{0}\right) & =0, \\
\phi_{r+1}\left(\tau_{\bar{N}}\right) & =1, \\
\phi_{r+1}\left(\tau_{0}\right) & =0 .
\end{aligned}
$$

In (35) $A$ is a $(4 \bar{N}+4) \times(4 \bar{N}+4)$ square matrix and $X$ and $R$ are $(4 \bar{N}+1) \times 1$ column vectors defined by

$$
\begin{aligned}
A & =\left[\begin{array}{llll}
A_{11} & A_{12} & A_{13} & A_{14} \\
A_{21} & A_{22} & A_{23} & A_{24} \\
A_{31} & A_{32} & A_{33} & A_{34} \\
A_{41} & A_{42} & A_{43} & A_{44}
\end{array}\right], \\
X & =\left[\begin{array}{l}
\mathbf{F}_{r+1} \\
\mathbf{G}_{r+1} \\
\Theta_{r+1} \\
\Phi_{r+1}
\end{array}\right], \\
R & =\left[\begin{array}{l}
\mathbf{R}_{1} \\
\mathbf{R}_{2} \\
\mathbf{R}_{3} \\
\mathbf{R}_{4}
\end{array}\right],
\end{aligned}
$$

where

$$
\begin{aligned}
\mathbf{F}= & {\left[f_{r+1}\left(\tau_{0}\right), f_{r+1}\left(\tau_{1}\right), \ldots, f_{r+1}\left(\tau_{\bar{N}}\right)\right]^{T}, } \\
\mathbf{G}= & {\left[g_{r+1}\left(\tau_{0}\right), g_{r+1}\left(\tau_{1}\right), \ldots, g_{r+1}\left(\tau_{\bar{N}}\right)\right]^{T}, } \\
\Theta= & {\left[\theta_{r+1}\left(\tau_{0}\right), \theta_{r+1}\left(\tau_{1}\right), \ldots, \theta_{r+1}\left(\tau_{\bar{N}}\right)\right]^{T}, } \\
\Phi= & {\left[\phi_{r+1}\left(\tau_{0}\right), \phi_{r+1}\left(\tau_{1}\right), \ldots, \phi_{r+1}\left(\tau_{\bar{N}}\right)\right]^{T}, } \\
A_{11}= & \left(\frac{1}{1-N}\right) \mathbf{D}^{3}+\operatorname{diag}\left[a_{1, r}\right] \mathbf{D}^{2}+\operatorname{diag}\left[a_{2, r}\right] \mathbf{D} \\
& +\operatorname{diag}\left[a_{3, r}\right], \\
A_{12}= & \left(\frac{N}{1-N}\right) \mathbf{D}, \\
A_{13}= & \mathbf{I}, \\
A_{14}= & \mathscr{B} \mathbf{I}, \\
A_{21}= & -\left(\frac{N}{1-N}\right) \mathbf{D}^{2}+\operatorname{diag}\left[b_{1, r}\right] \mathbf{D}+\operatorname{diag}\left[b_{2, r}\right], \\
A_{22}= & \left(\frac{2-N}{2-2 N}\right) \mathbf{D}^{2}+\operatorname{diag}\left[b_{3, r}\right] \mathbf{D}+\operatorname{diag}\left[b_{4, r}\right], \\
A_{23}= & \mathbf{0}, \\
A_{24}= & \mathbf{0}, \\
A_{31}= & \operatorname{diag}\left[c_{1, r}\right], \\
A_{32}= & \mathbf{0}, \\
A_{33}= & \frac{1}{\operatorname{Pr}} \mathbf{D}^{2}+\operatorname{diag}\left[c_{2, r}\right] \mathbf{D},
\end{aligned}
$$

TABLE 1: Comparison of $-\theta^{\prime}(0)$ for free convection along a vertical flat plate in Newtonian fluid when $N=0, n=0, \mathscr{B}=0, \operatorname{Pr}=1$, $\mathrm{Bi} \rightarrow \infty$, and $f_{w}=0$.

\begin{tabular}{lccc}
\hline Merkin [36] & Nazar et al. [37] & Molla et al. [38] & Present \\
\hline 0.4214 & 0.4214 & 0.4214 & 0.4214313 \\
\hline
\end{tabular}

$$
\begin{aligned}
& A_{34}=\mathbf{0}, \\
& A_{41}=\operatorname{diag}\left[d_{1, r}\right], \\
& A_{42}=\mathbf{0}, \\
& A_{43}=\mathbf{0}, \\
& A_{44}=\frac{1}{\operatorname{Sc}} \mathbf{D}^{2}+\operatorname{diag}\left[d_{2, r}\right] \mathbf{D}, \\
& \mathbf{R}_{1}=R_{1, r}, \\
& \mathbf{R}_{2}=R_{2, r}, \\
& \mathbf{R}_{3}=R_{3, r}, \\
& \mathbf{R}_{4}=R_{4, r},
\end{aligned}
$$

and here $\mathbf{I}$ is an identity matrix, the size of the matrix $\mathbf{0}$ is $(\bar{N}+1) \times 1$, and diag [ ] is a diagonal matrix of size $(\bar{N}+1) \times(\bar{N}+$ 1). Subscript $\mathbf{r}$ denotes the iteration number. After modifying matrix system (35) to incorporate boundary condition (36), the solution is obtained as

$$
X=A^{-1} R .
$$

\section{Results and Discussions}

It is noticed that the present problem reduces to free convection heat transfer along an impermeable vertical plate in a micropolar fluid without the convective boundary condition when $f_{w}=0, \mathrm{Bi} \rightarrow \infty$, and $\mathscr{B}=0$. Also in the limit as $N \rightarrow$ 0 , governing equations (2)-(5) reduce to the corresponding equations for a free convection heat and mass transfer in a viscous fluid. In order to validate the code generated the results of the present problem have been compared with the results obtained by Merkin [36], Nazar et al. [37], and Molla et al. [38] as a special case by taking $N=0, n=0, \mathscr{B}=0$, $\operatorname{Pr}=1, \mathrm{Bi} \rightarrow \infty$, and $f_{w}=0$ and it was found that they are in good agreement, as presented in Table 1. Also, the comparison of heat transfer coefficient has been made with the results obtained by Nazar et al. [37] as shown in Table 2 when $n=0.5, \mathscr{B}=0, \operatorname{Pr}=1, \mathrm{Bi} \rightarrow \infty$, and $f_{w}=0$. To study the effects of coupling number $N$, suction/injection parameter $f_{w}$, Biot number $\mathrm{Bi}$, and material parameter $n$, computations were carried out in the cases of $\mathscr{B}=1.0$, $\operatorname{Pr}=0.71$, and $\mathrm{Sc}=0.22$.

The effects of the coupling number $N$ on the dimensionless velocity, microrotation, temperature, and concentration are illustrated in Figures 2(a)-2(d) with fixed values of other parameters. The coupling number $N$ characterizes the coupling of linear and rotational motion arising from the 


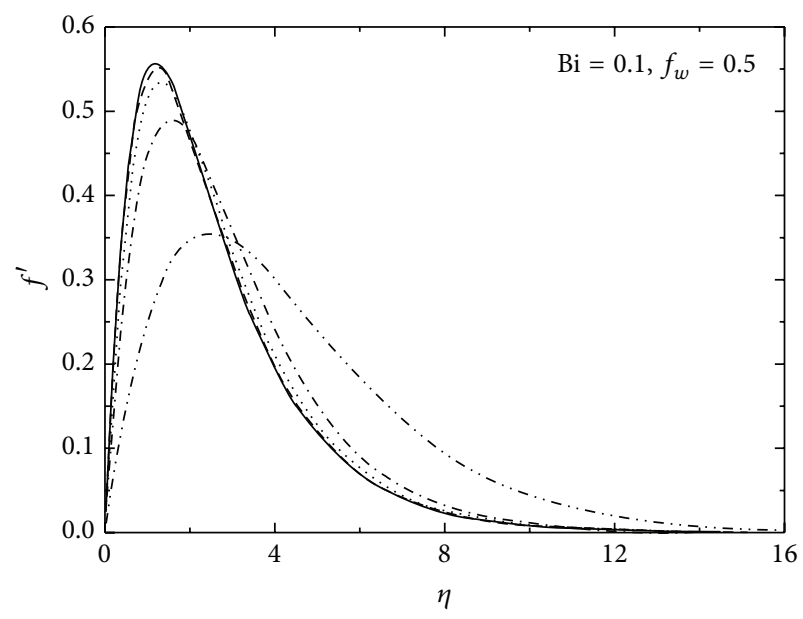

$\begin{aligned} n & =0.0, N=0.0 \\ ---n & =0.5, N=0.1 \\ \cdots \cdots n & =0.5, N=0.3\end{aligned}$

(a)

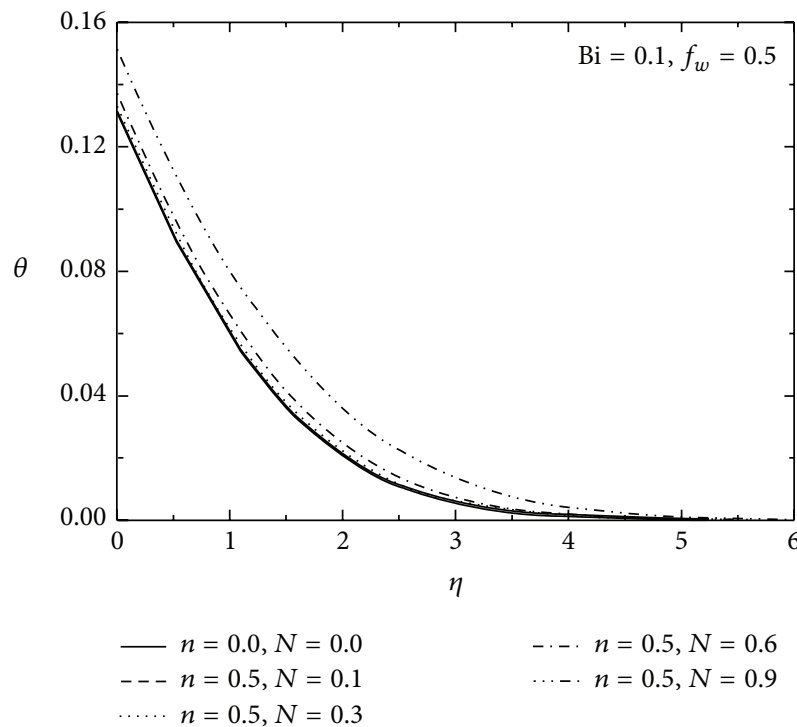

(c)

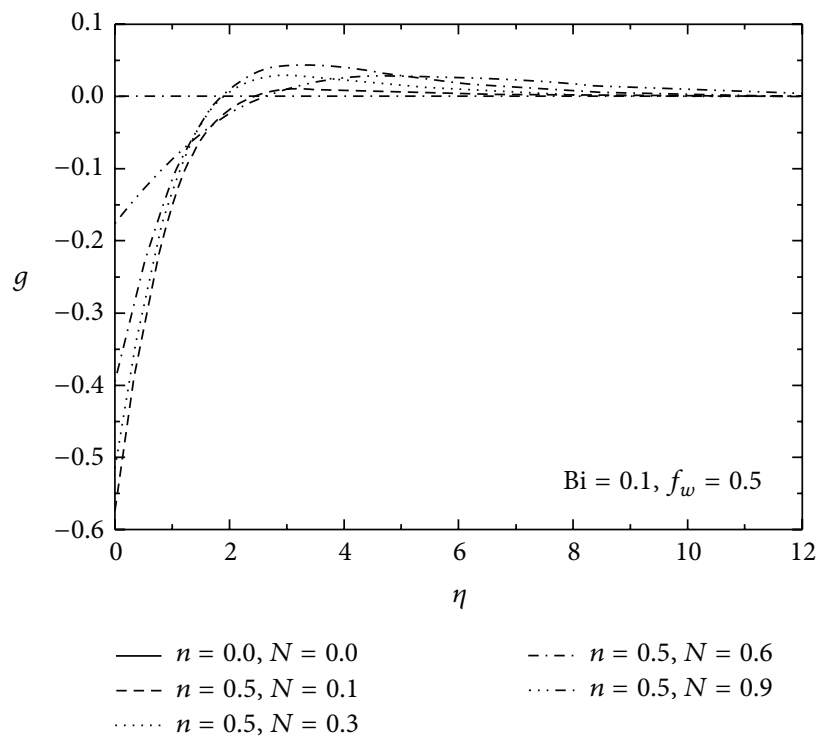

(b)

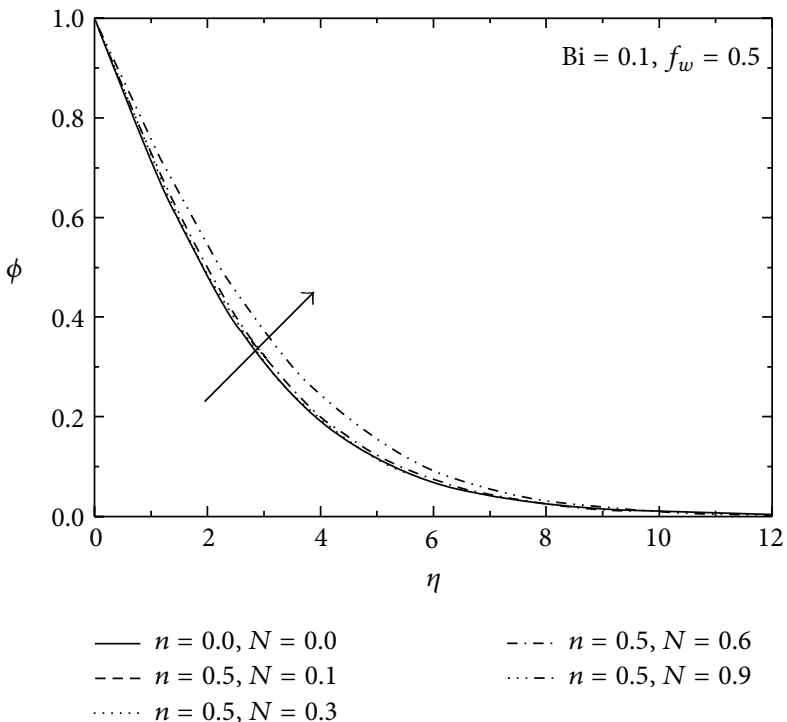

(d)

FIGURE 2: Effect of $N$ on (a) velocity, (b) microrotation, (c) temperature, and (d) concentration profiles.

TABle 2: Comparison of $-\theta^{\prime}(0)$ for free convection flow in a micropolar fluid obtained by Nazar et al. [37] when $n=0.5, \mathscr{B}=0$, $\operatorname{Pr}=1, \mathrm{Bi} \rightarrow \infty$, and $f_{w}=0$.

\begin{tabular}{lcc}
\hline$N$ & Nazar et al. [37] & Present \\
\hline 0.00 & 0.4214 & 0.4214 \\
0.33 & 0.3991 & 0.3990 \\
0.50 & 0.3834 & 0.3834 \\
0.60 & 0.3709 & 0.3709 \\
0.66 & 0.3608 & 0.3608 \\
0.71 & 0.3522 & 0.3522 \\
0.75 & 0.3447 & 0.3447 \\
\hline
\end{tabular}

fluid particles. In the case of $N=0$ (i.e., as $\kappa$ tends to zero) the micropolarity is absent and fluid becomes nonpolar fluid. With a large value of $N$ effect of microstructure becomes significant, whereas with a diminished value of $N$ the individuality of the substructure is much less articulated. As $N$ increases, it is found from Figure 2(a) that the maximum velocity decreases in amplitude and the location of the maximum velocity moves farther away from the wall. Since $N \rightarrow 0$ corresponds to viscous fluid, the velocity in case of a micropolar fluid has been less compared to that of viscous fluid case. It can be observed from Figure 2(b) that, as $N$ increases, initially microrotation profiles tend to become flatter and then approach their free stream values far away from the wall. This happens due to the vanishing 

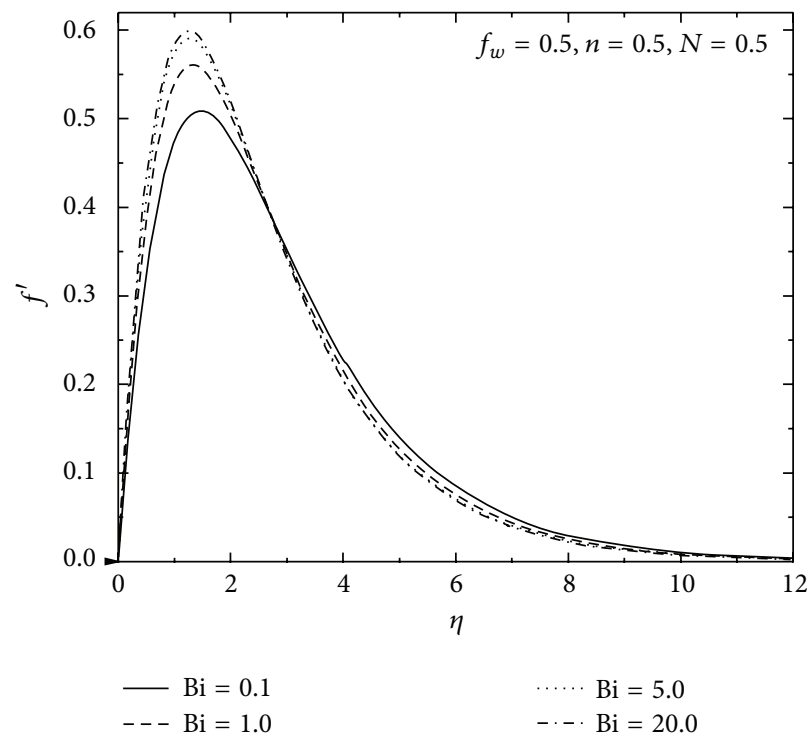

(a)

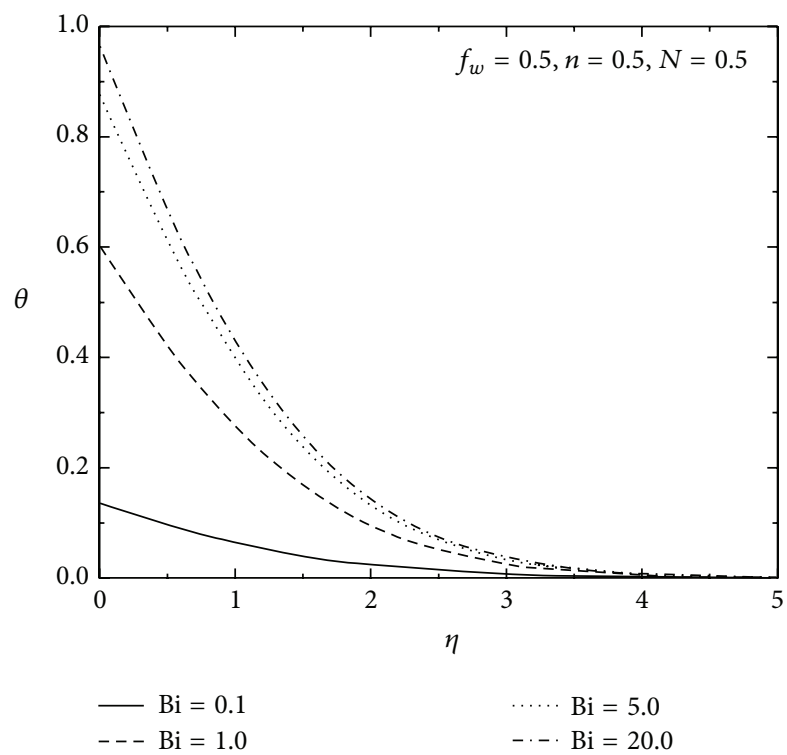

(c)

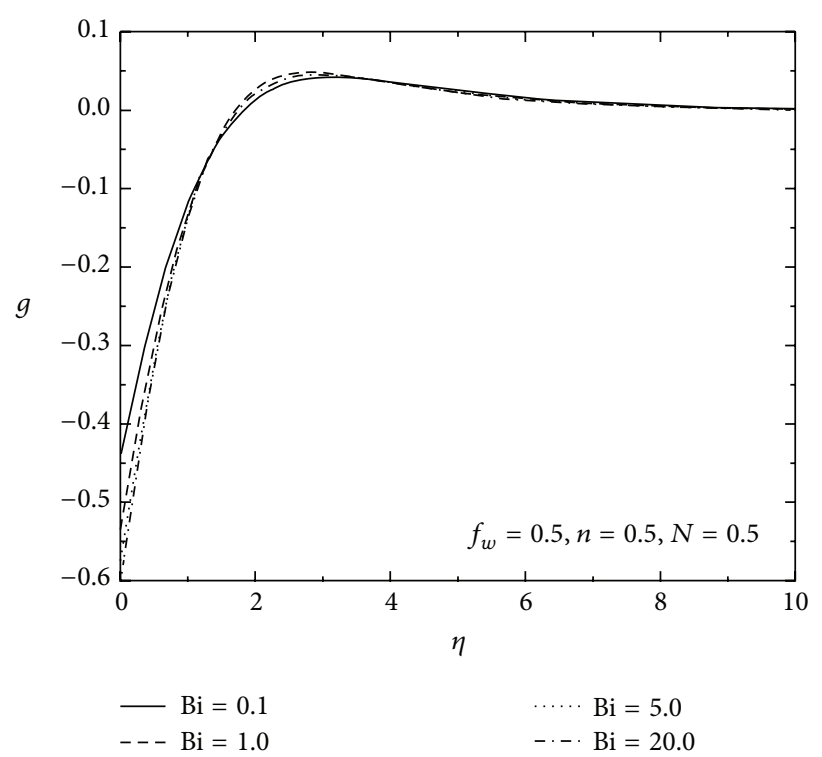

(b)

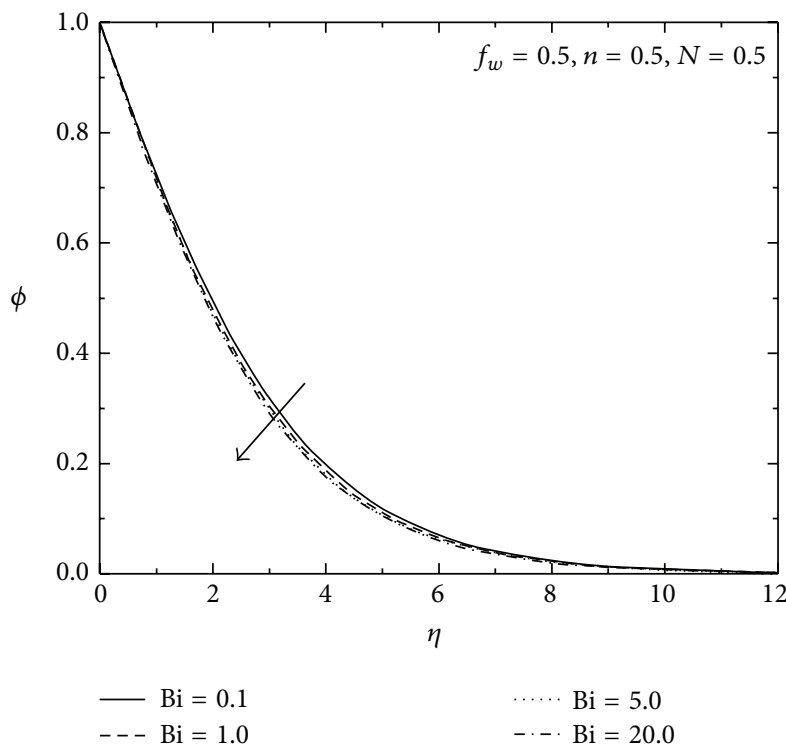

(d)

FIGURE 3: Effect of Bi on (a) velocity, (b) microrotation, (c) temperature, and (d) concentration profiles.

of antisymmetric part of the stress on the boundary that corresponds to a weak concentration of microelements. This is because an increment in the value of $N$ implies a higher vortex viscosity of fluid which promotes the microrotation of micropolar fluids. It is seen from Figures 2(c) and 2(d) that thermal and concentration boundary layers of the fluid increase with increase in coupling number $N$. Hence, temperature and concentration in case of micropolar fluids are more than those of the viscous fluid case.

The Biot number $\mathrm{Bi}$ is the ratio of internal thermal resistance of a solid to boundary layer thermal resistance. When $\mathrm{Bi}=0$ the plate is totally insulated, internal thermal resistance of the plate is extremely high, and no convective heat transfer to the cold fluid on the upper part of the plate takes place. Figure 3(a) depicts fluid velocity profiles for different values of the Biot number with $N=0.5, f_{w}=$ 0.5 , and $n=0.5$. Generally, fluid velocity is zero at plate surface and increases gradually away from plate to free stream value satisfying boundary conditions. It is interesting to note that an increase in the intensity of convective surface heat transfer Bi produces significant enhancement in fluid velocity within the momentum boundary layer. In Figure 3(b), we bring out the behavior of microrotation with different values of Biot number Bi for fixed values of other parameters. As the parameter value $B i$ increases microrotation showing reverse rotation near the two boundaries. Hence, the condition of vanishing of antisymmetric part of the stress on the boundary results in a drastic change of the microrotation profiles. 

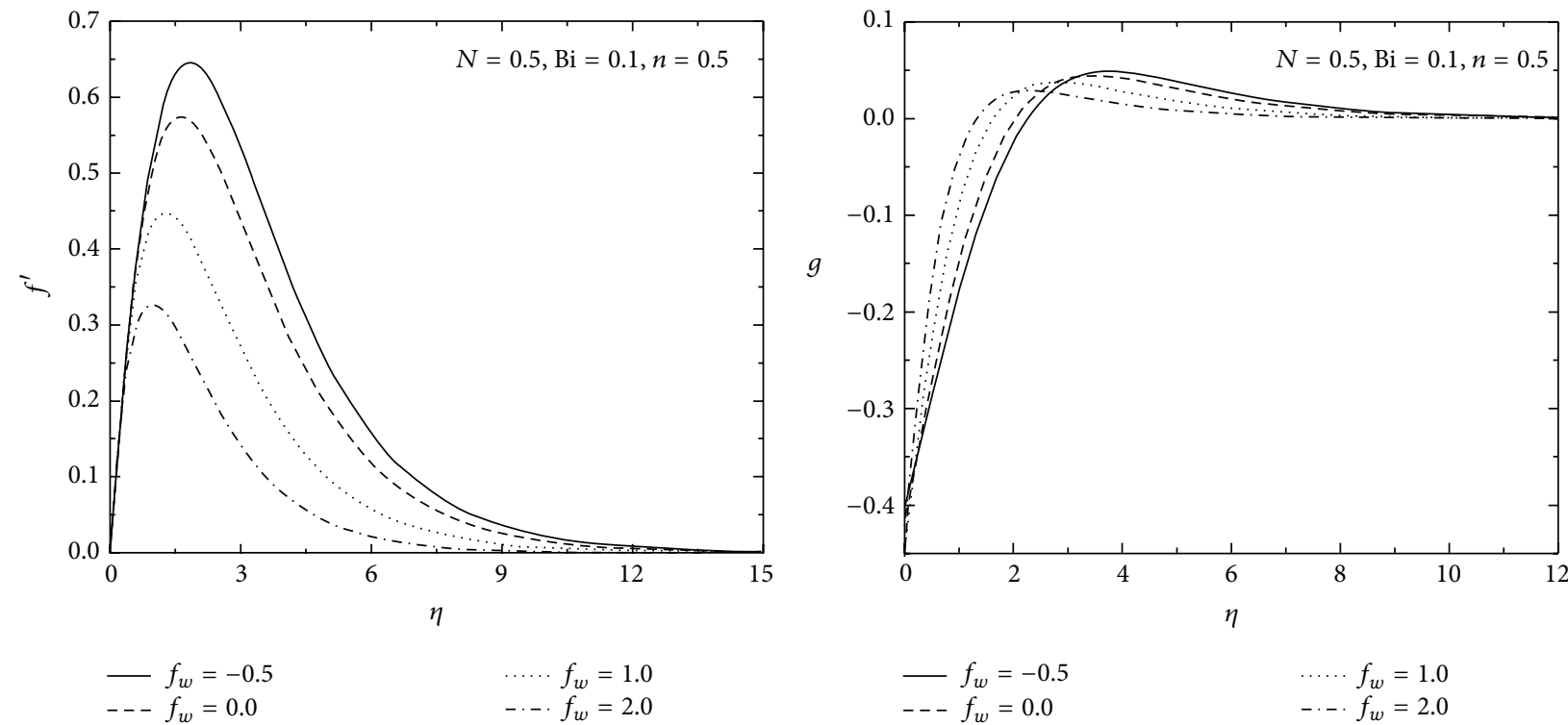

(a)

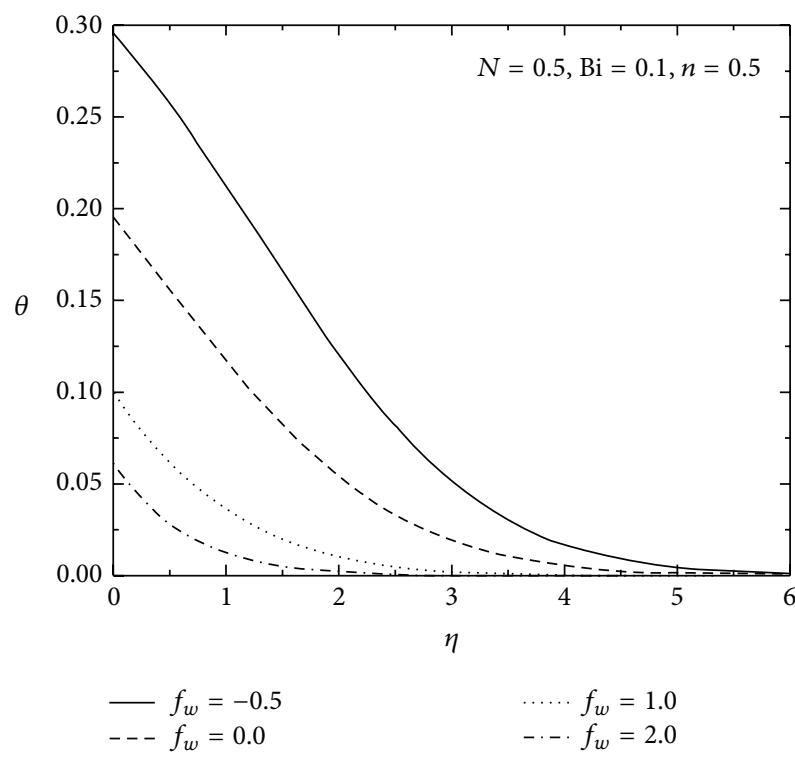

(c)

(b)

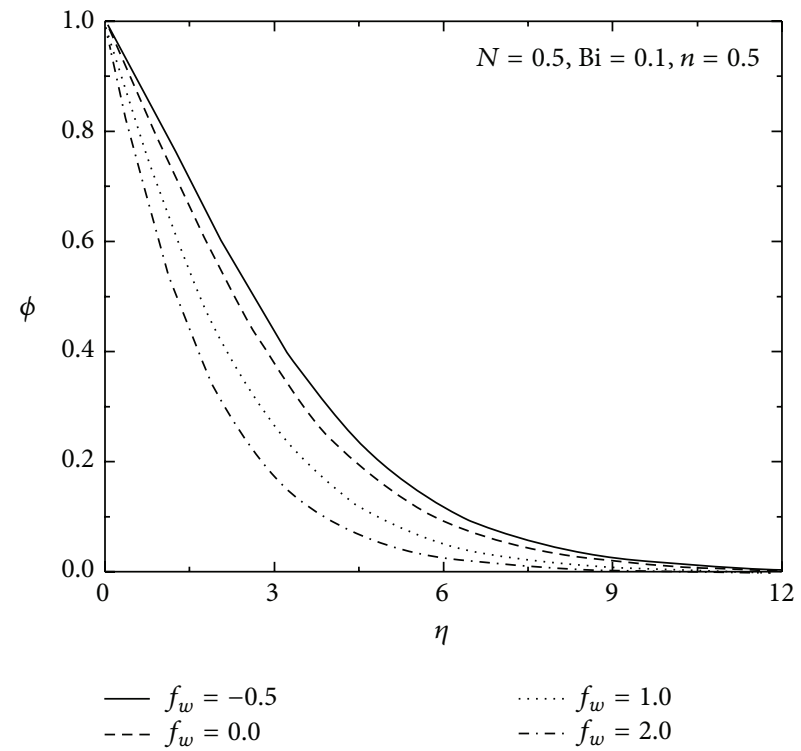

(d)

FIgURE 4: Effect of $f_{w}$ on (a) velocity, (b) microrotation, (c) temperature, and (d) concentration profiles.

Given that convective heating increases with Biot number, $\mathrm{Bi} \rightarrow \infty$ simulates the isothermal surface which is clearly seen from Figure 3(c), where $\theta(0)=1$ as $\mathrm{Bi} \rightarrow \infty$. In fact, a high Biot number indicates higher internal thermal resistance of the plate than boundary layer thermal resistance. In this fluid temperature is maximum at the plate surface and decreases exponentially to zero value far out from the plate satisfying boundary conditions. As a consequence, an increment in the Biot number leads to increase of fluid temperature efficiency. Figure 3(d) illustrates the variation of dimensionless concentration for different values of $\mathrm{Bi}$. It is clear that the concentration of fluid decreases with increase of Bi.
The effect of $f_{w}$ on velocity profile is depicted in Figure 4(a). Here, $f_{w}>0$ represents suction and $f_{w}<0$ denotes injection. The lower velocity is noticed in case of suction when compared to case of injection. From Figure 4(b), we note that microrotation is showing reverse rotation near two boundaries with both suction and injection parameter. The dimensionless temperature for different values of suction/injection parameters is drawn in Figure 4(c). It is readable that the temperature of the fluid is more in case of injection, whereas it is less in case of suction in comparison with the impermeable surface case $\left(f_{w}=0\right)$. Figure $4(\mathrm{~d})$ demonstrates dimensionless concentration for different values of suction/injection parameters. It is determined that the 


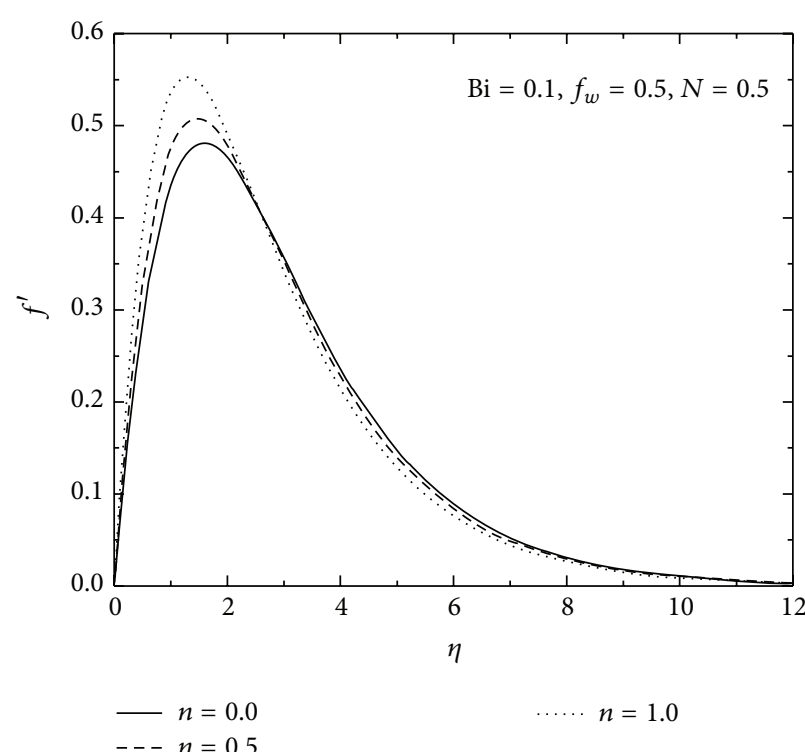

(a)

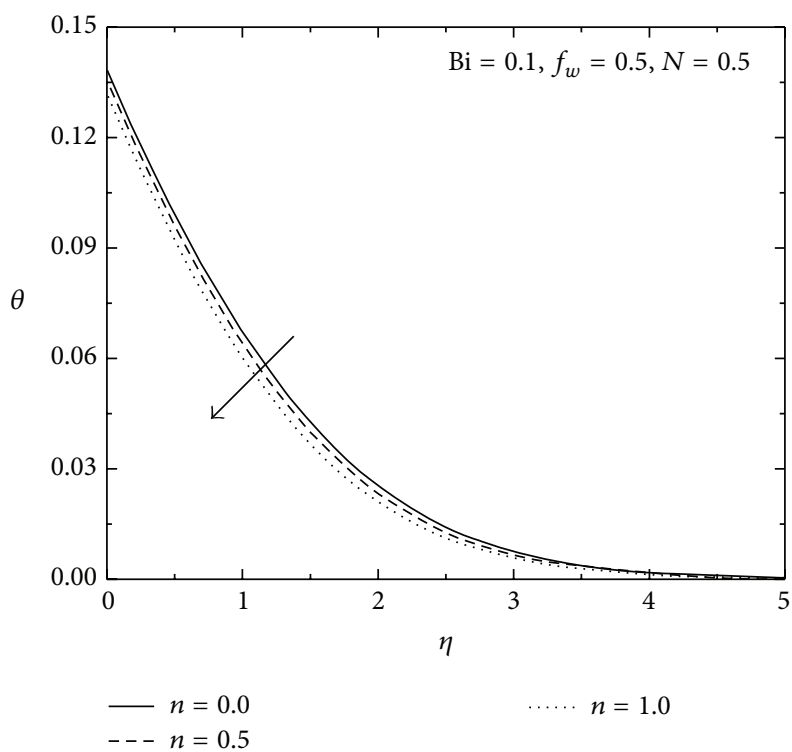

(c)

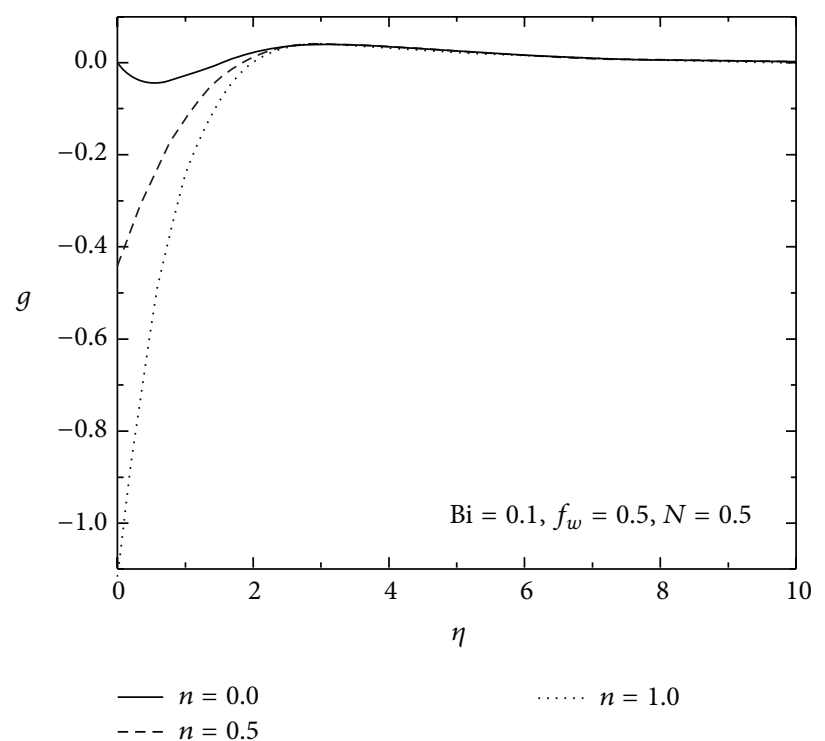

(b)

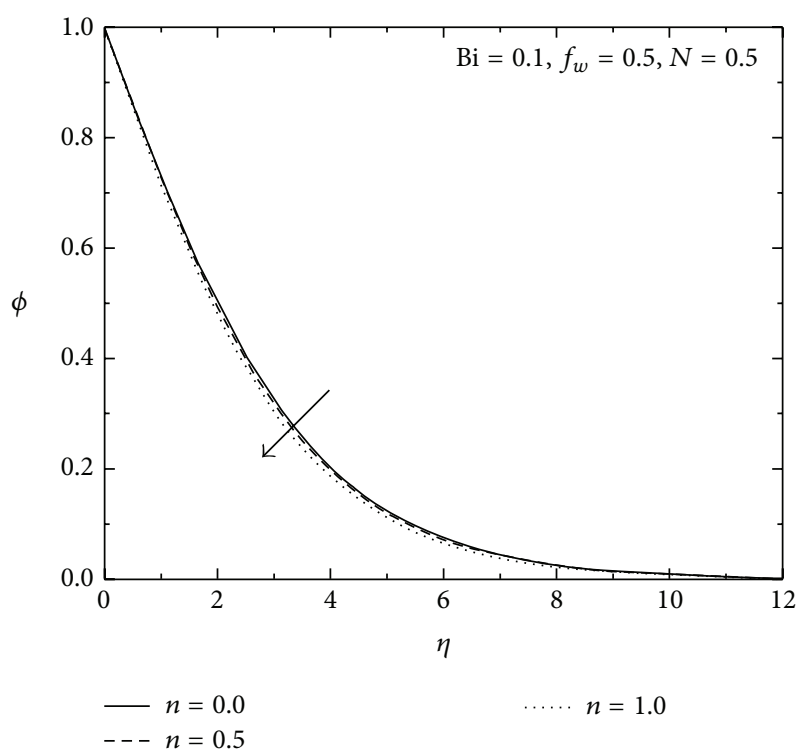

(d)

FIGURE 5: Effect of material parameter $n$ on (a) velocity, (b) microrotation, (c) temperature, and (d) concentration profiles.

concentration of fluid is more with injection, whereas it is less with suction when compared to the impermeable surface case $\left(f_{w}=0\right)$. As a finale, the thermal and solutal boundary layer thicknesses increase in case of injection compared to case of suction as displayed in Figures 4(c) and 4(d).

In Figures 5(a)-5(d), the effect of material parameter $n$ on dimensionless velocity, microrotation, temperature, and concentration is presented for fixed values of other parameters, since the material parameter $n$ signifies the microrotation effects (i.e., for $n=0$, particles are not free to revolve near the surface whereas, as $n$ increases from 0 to 1 , the microrotation term gets augmented and induces flow enhancement). As $n$ increases, it is found from Figure 5(a) that the minimum velocity increases in amplitude and the location of the minimum velocity moves farther away from the wall. From Figure 5(b), we observe that the microrotation is decreasing with increasing value of material parameter $n$ within the boundary layer. It is clear from Figures 5(c)5(d) that the thermal and solutal boundary layer thicknesses decrease with increase of material parameter $n$.

The variations of $-\theta^{\prime}(0)$ and $-\phi^{\prime}(0)$ versus coupling number $N$ are shown in Figures 6-8. It can be noticed from these figures that the heat and mass transfer coefficients are less in case of micropolar fluids when compared to the viscous fluids. This is because as $N$ increases, the thermal and solutal boundary layer thicknesses become larger, thus giving rise to a small value of local heat and mass transfer rates. The effect of the Biot number Bi with fixed $f_{w}=0.5$ 

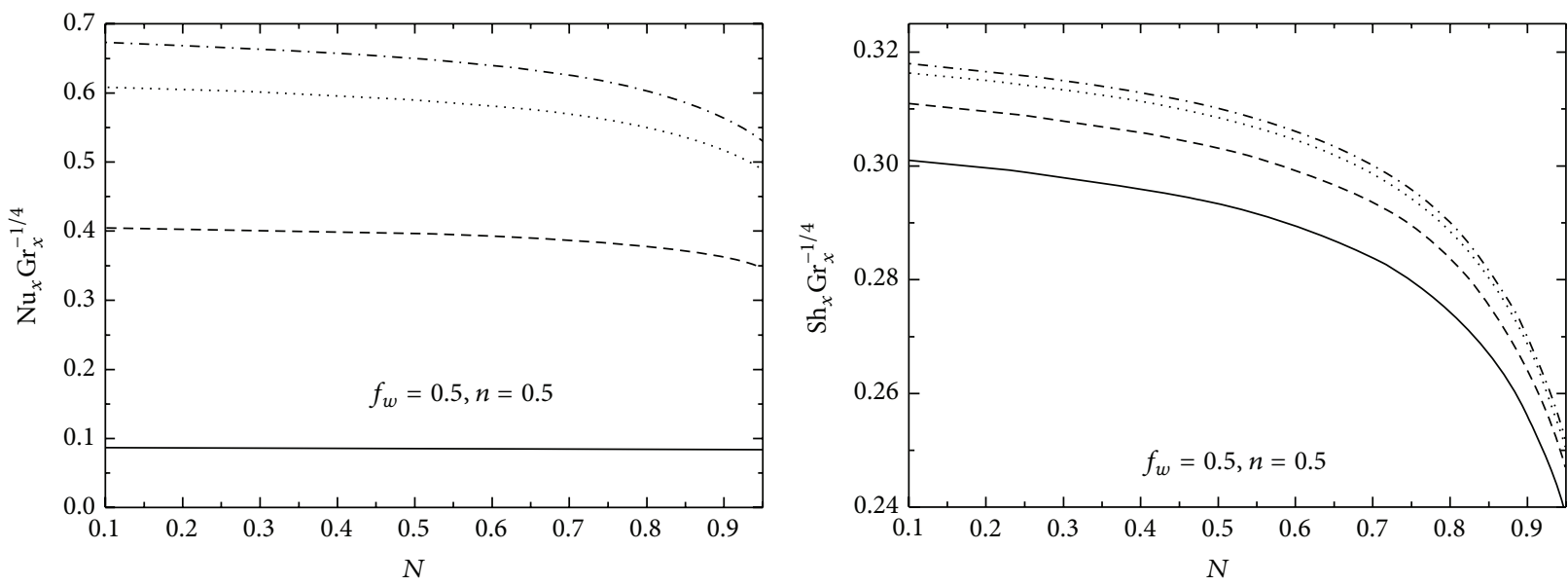

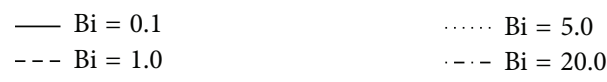

(a)
$\begin{aligned}-\mathrm{Bi} & =0.1 \\ ---\mathrm{Bi} & =1.0\end{aligned}$

(b)

FIGURE 6: Effect of Bi on (a) heat transfer rate and (b) mass transfer rate.

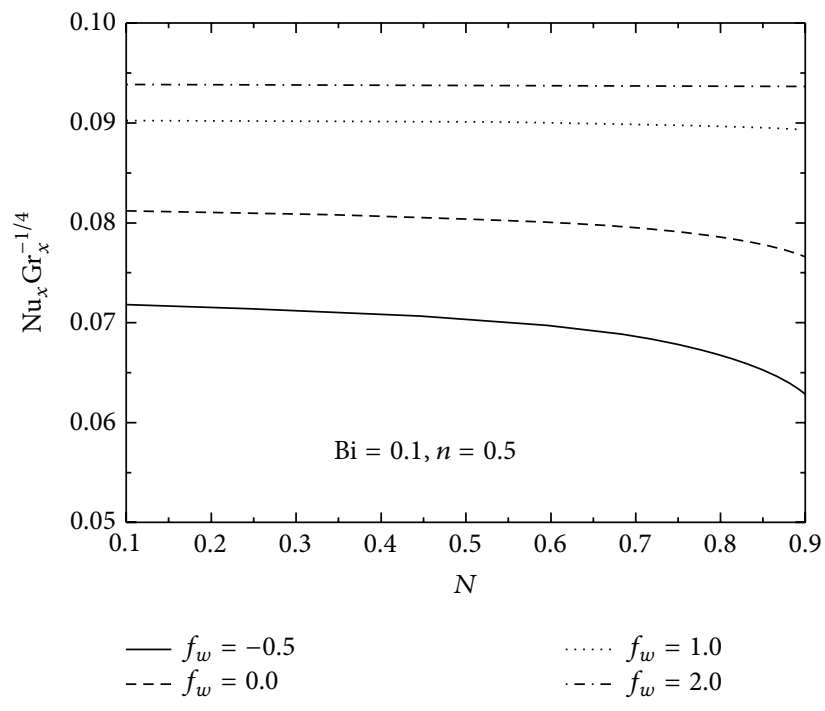

(a)

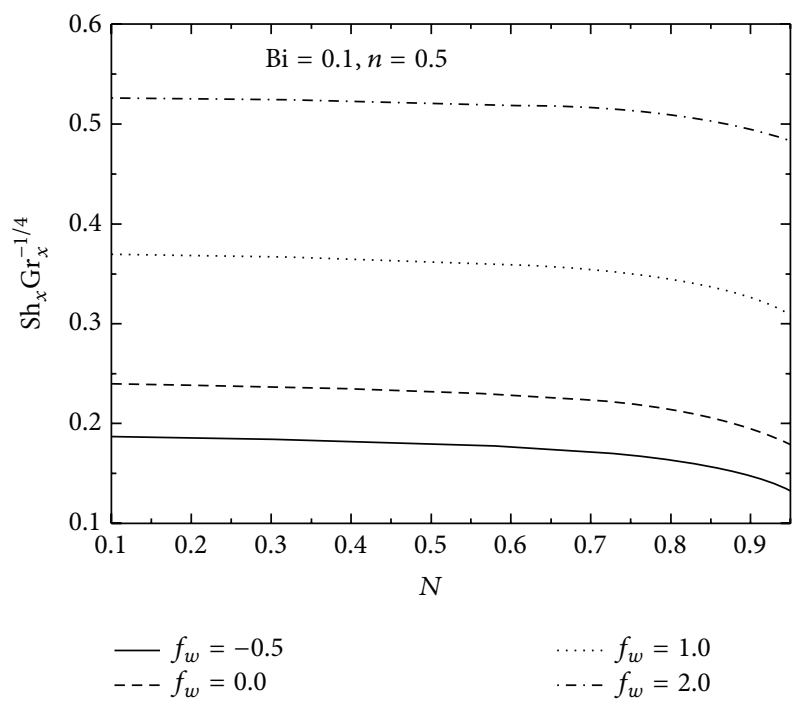

(b)

FIGURE 7: Effect of $f_{w}$ on (a) heat transfer rate and (b) mass transfer rate.

and $n=0.5$ on local heat transfer coefficient is exhibited in Figure 6. It is found from Figure 6 that local heat transfer rate increases nonlinearly with the increase in Biot number $\mathrm{Bi}$. The influence of the Biot number $\mathrm{Bi}$ on local mass transfer coefficient is shown in Figure 6. Figure 6 reveals that the local mass transfer coefficient is enhanced by the growth in the Biot number Bi. In Figure 7, the effect of the suction/injection parameter $f_{w}$ with fixed $\mathrm{Bi}=0.1$ and $n=0.5$ on local heat and mass transfer coefficients is displayed. It is found from Figure 7 that the local heat and mass transfer coefficients are less in the case of injection $f_{w}<0$ in comparison with the case of suction $f_{w}>0$. Figure 8 is prepared to analyze the effect of the material parameter $n$ with fixed $\mathrm{Bi}=0.1$ and $f_{w}=0.5$ on local heat and mass transfer coefficients. Figure 8 reveals that the local heat and mass transfer coefficients are enhanced by the increase in material parameter $n$. This is because when $n$ increases from 0 to 1 , the microrotation term gets augmented and induces flow enhancement.

The variations of $C_{f} \mathrm{Gr}_{\bar{x}}^{1 / 4}$ and $M_{w} \mathrm{Gr}_{\bar{x}}^{1 / 2}$, which are proportional to the coefficients of skin friction and wall couple stress, are shown in Table 3 with different values of the coupling number $N$ for fixed $n=0.5, f_{w}=0.5$, and $\mathrm{Bi}=0.1$. It indicates that the skin friction factor is higher for micropolar fluid than the viscous fluids $(N=0)$. Since micropolar fluids offer a heavy resistance (resulting from vortex viscosity) to fluid movement and cause larger skin 


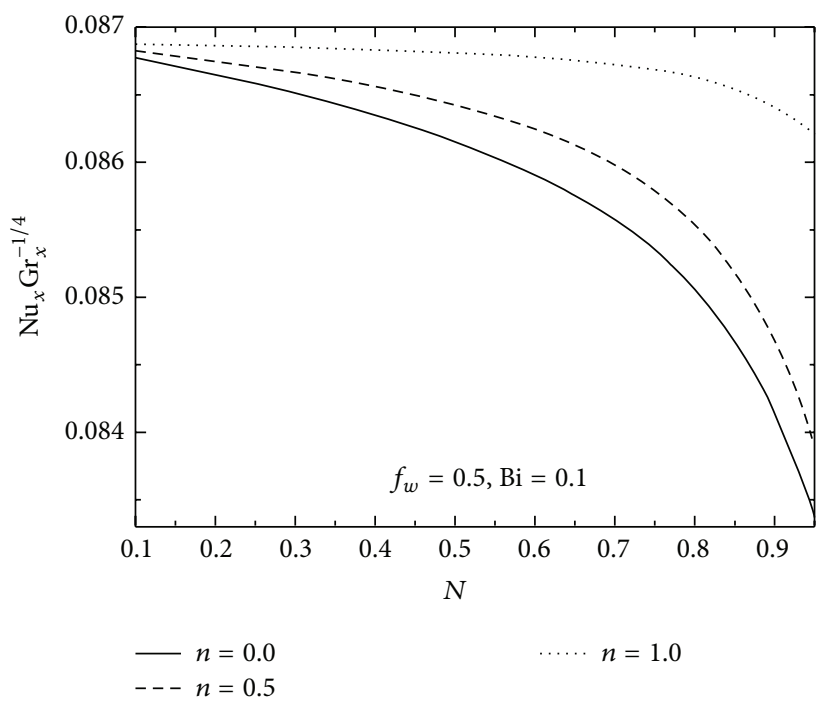

(a)

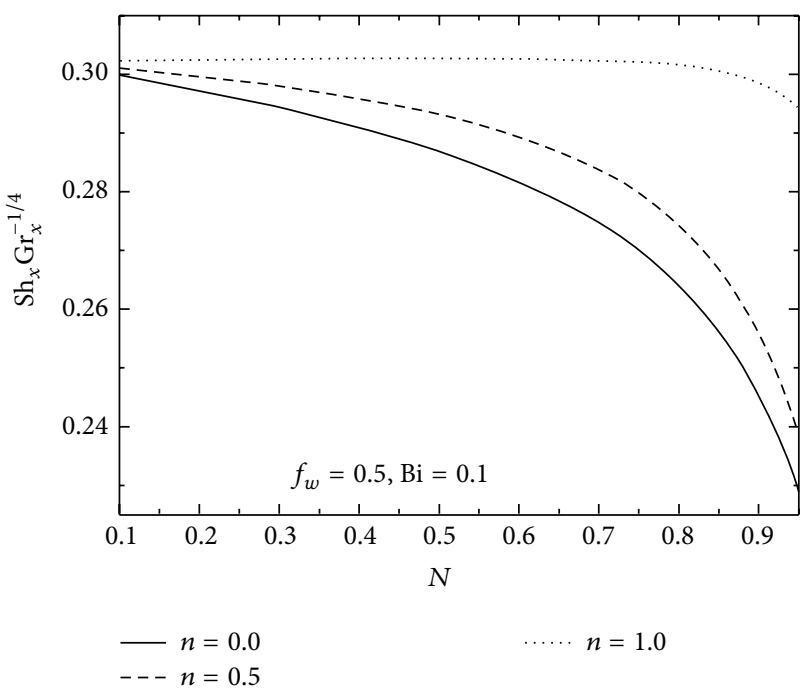

(b)

FIGURE 8: Effect of material parameter $n$ on (a) heat transfer rate and (b) mass transfer rate.

TABLE 3: Effects of skin friction and wall couple stress for varying values of Biot numbers $\mathrm{Bi}$, micropolar parameter $N$, material parameter $n$, and suction/injection parameter $f_{w}$.

\begin{tabular}{cccccc}
\hline$N$ & $\mathrm{Bi}$ & $f_{w}$ & $n$ & $C_{f} \mathrm{Gr}_{\bar{x}}^{1 / 4}$ & $M_{w} \mathrm{Gr}_{\bar{x}}^{1 / 2}$ \\
\hline 0.1 & 0.1 & 0.5 & 0.5 & 2.424227 & 0.63279 \\
0.3 & 0.1 & 0.5 & 0.5 & 2.502716 & 0.639966 \\
0.5 & 0.1 & 0.5 & 0.5 & 2.647987 & 0.630863 \\
0.7 & 0.1 & 0.5 & 0.5 & 2.943561 & 0.603879 \\
0.9 & 0.1 & 0.5 & 0.5 & 3.874631 & 0.550891 \\
\hline 0.5 & 0.1 & 0.5 & 0.5 & 2.647987 & 0.630863 \\
0.5 & 1.0 & 0.5 & 0.5 & 3.211755 & 0.805879 \\
0.5 & 5.0 & 0.5 & 0.5 & 3.533141 & 0.908078 \\
0.5 & 20.0 & 0.5 & 0.5 & 3.629791 & 0.939137 \\
\hline 0.5 & 0.1 & -0.5 & 0.5 & 2.426265 & 0.336219 \\
0.5 & 0.1 & 0.0 & 0.5 & 2.530014 & 0.465429 \\
0.5 & 0.1 & 1.0 & 0.5 & 2.737553 & 0.820899 \\
0.5 & 0.1 & 2.0 & 0.5 & 2.721244 & 1.193596 \\
\hline 0.5 & 0.1 & 0.5 & 0.0 & 2.916655 & -0.289339 \\
0.5 & 0.1 & 0.5 & 0.5 & 2.647987 & 0.630863 \\
0.5 & 0.1 & 0.5 & 1.0 & 2.232556 & 2.066191 \\
\hline
\end{tabular}

friction factor compared to viscous fluid, the results as well suggest larger values of coupling number $N$ and lower wall couple stresses. Since the skin friction coefficient $f^{\prime \prime}(0)$ and wall couple stress coefficient as well as high temperature and mass transport rates are more depressed in the micropolar fluid comparing to the viscous fluid, which may be beneficial in flow, temperature, and concentration control of polymer processing, thus, the presence of microscopic effects arising from the local structure and of the fluid elements reduces the high temperature and mass transfer coefficients. The effect of $\mathrm{Bi}$ on $C_{f} \mathrm{Gr}_{\bar{x}}^{1 / 4}$ and $M_{w} \mathrm{Gr}_{\bar{x}}^{1 / 2}$ for $f_{w}=0.5, n=0.5$, and
$N=0.5$ is illustrated in Table 3. It can be noticed that the skin friction and wall couple stress coefficients are increasing with increase of $\mathrm{Bi}$ for fixed values of other parameters. This notice is consistent with physical profiles presented in Figure 3. The effects of suction/injection parameter on the skin friction and wall couple stress coefficients are also shown in Table 3. It is noted that the skin friction and wall couple stress coefficients are less with injection case, whereas they are more with suction case when compared to the case of impermeable surface. Finally, the detailed behavior of the material parameter $n$ is given in Table 3. The skin friction decreases and wall couple stress increases with increase of material parameter $n$.

\section{Conclusions}

In this composition, the similarity solution of the free convection flow on a permeable vertical plate of a micropolar fluid under the convective boundary condition is obtained using Lie group transformations. Using the similarity variables, the governing equations are transformed into a set of nondimensional parabolic equations. These equations are solved numerically using spectral quasi-linearisation method. The numerical computation is carried out for various values of nondimensional physical parameters. The main findings are summarized as follows:

(i) The numerical results indicate that velocity distribution is less near the plate but it is more far away from the plate; the wall couple stress coefficient and rate of heat and mass transfers are lower but the temperature and concentration distributions and the skin friction coefficient are higher for the micropolar fluids in comparison with those of viscous fluids. Also, the reverse rotation of microrotation near two boundaries is found with the increasing value of $N$. 
(ii) An increase in Biot number Bi decreases concentration distribution, whereas it causes an increase in temperature distribution, skin friction and wall couple stress coefficients, and heat and mass transfer rates within the boundary layer. Further, enhancing in Biot number $\mathrm{Bi}$ enhances velocity distribution near the plate but shows the reverse behavior far away from the plate. We observe reverse rotation of microrotation near two boundaries within the boundary layer in the presence of Biot number.

(iii) Less velocity, temperature, and concentration distributions are observed, more skin friction and wall couple stress coefficients and heat and mass transfer rates in the case of suction compared to the case of injection. Further, microrotation decreases near the wall and depicts the opposite trend far away from the wall.

(iv) It is found that microrotation, temperature, and concentration distributions and skin friction coefficients are more in the case of a micropolar fluid with strong concentration (i.e., $n=0$ ) when compared to the case of a micropolar fluid with weak concentration (i.e., $n=1 / 2)$. Further, velocity is less in the case of $n=0$ when compared to the case of $n=1 / 2$ near the wall and shows the opposite trend far away from the wall.

\section{Conflict of Interests}

The authors declare that there is no conflict of interests regarding the publication of this paper.

\section{Acknowledgment}

The authors are thankful to the reviewers for their valuable suggestions and comments.

\section{References}

[1] J. H. Merkin, "Natural-convection boundary-layer flow on a vertical surface with Newtonian heating," International Journal of Heat and Fluid Flow, vol. 15, no. 5, pp. 392-398, 1994.

[2] A. Aziz, "A similarity solution for laminar thermal boundary layer over a flat plate with a convective surface boundary condition," Communications in Nonlinear Science and Numerical Simulation, vol. 14, no. 4, pp. 1064-1068, 2009.

[3] O. D. Makinde, "Similarity solution for natural convection from a moving vertical plate with internal heat generation and a convective boundary condition," Thermal Science, vol. 15, pp. S137-S143, 2011.

[4] S. M. Ibrahim and N. Bhashar Reddy, "Similarity solution of heat and mass transfer for natural convection over a moving vertical plate with internal heat generation and a convective boundary condition in the presence of thermal radiation, viscous dissipation, and chemical reaction," ISRN Thermodynamics, vol. 2013, Article ID 790604, 10 pages, 2013.

[5] C. RamReddy, P. V. S. N. Murthy, A. J. Chamkha, and A. M. Rashad, "Soret effect on mixed convection flow in a nanofluid under convective boundary condition," International Journal of Heat and Mass Transfer, vol. 64, pp. 384-392, 2013.
[6] H. T. Alkasasbeh, M. Z. Salleh, R. M. Tahar, and R. Nazar, "Numerical solutions of free convection boundary layer flow on a solid sphere with convective boundary conditions," Journal of Physics: Conference Series, vol. 495, Article ID 012025, 2014.

[7] A. Pantokratoras, "A note on natural convection along a convectively heated vertical plate," International Journal of Thermal Sciences, vol. 76, pp. 221-224, 2014.

[8] G. Lukaszewicz, Micropolar Fluids-Theory and Applications, Birkhäauser, Basel, Switzerland, 1999.

[9] V. A. Eremeyev, L. P. Lebedev, and H. Altenbach, Foundations of Micropolar Mechanics, Springer, New York, NY, USA, 2013.

[10] G. Ahmadi, "Self-similar solution of imcompressible micropolar boundary layer flow over a semi-infinite plate," International Journal of Engineering Science, vol. 14, no. 7, pp. 639-646, 1976.

[11] D. A. Rees and I. Pop, "Free convection boundary-layer flow of a micropolar fluid from a vertical flat plate," IMA Journal of Applied Mathematics, vol. 61, no. 2, pp. 179-197, 1998.

[12] D. Srinivasacharya and C. RamReddy, "Effect of double stratification on mixed convection in a micropolar fluid," Matematika, vol. 28, no. 2, pp. 133-149, 2012.

[13] D. Srinivasacharya and C. Ramreddy, "Mixed convection heat and mass transfer in a doubly stratified micropolar fluid," Computational Thermal Sciences, vol. 5, no. 4, pp. 273-287, 2013.

[14] D. Srinivasacharya and C. RamReddy, "Natural convection heat and mass transfer in a micropolar fluid with thermal and mass stratification," International Journal for Computational Methods in Engineering Science and Mechanics, vol. 14, no. 5, pp. 401-413, 2013.

[15] N. A. Yacob and A. Ishak, "Stagnation point flow towards a stretching/shrinking sheet in a micropolar fluid with a convective surface boundary condition," The Canadian Journal of Chemical Engineering, vol. 90, no. 3, pp. 621-626, 2012.

[16] K. Zaimi and A. Ishak, "Stagnation-point flow and heat transfer over a nonlinearly stretching/shrinking sheet in a micropolar fluid," Abstract and Applied Analysis, vol. 2014, Article ID 261630, 6 pages, 2014.

[17] L. V. Ovsiannikov, Group Analysis of Differential Equations, Academic Press, New York, NY, USA, 1982.

[18] P. J. Olver, Applications of Lie Groups to Differential Equations, vol. 107 of Graduate Texts in Mathematics, Springer, New York, NY, USA, 2nd edition, 1993.

[19] G. W. Bluman and S. C. Anco, Symmetry and Integration Methods for Differential Equations, Springer, 2009.

[20] T. Tapanidis, G. Tsagas, and H. P. Mazumdar, "Application of scaling group of transformations to viscoelastic second-grade fluid flow," Nonlinear Functional Analysis and Applications, vol. 8, no. 3, pp. 345-350, 2003.

[21] I. A. A. Hassanien and M. A. Hamad, "Group theoretic method for unsteady free convection flow of a micropolar fluid along a vertical plate in a thermally stratified medium," Applied Mathematical Modelling. Simulation and Computation for Engineering and Environmental Systems, vol. 32, no. 6, pp. 1099-1114, 2008.

[22] R. Kandasamy, K. Gunasekaran, and S. B. H. Hasan, "Scaling group transformation on fluid flow with variable stream conditions," International Journal of Non-Linear Mechanics, vol. 46, no. 7, pp. 976-985, 2011.

[23] A. Aziz, M. J. Uddin, M. A. A. Hamad, and A. I. Md. Ismail, "MHD flow over an inclined radiating plate with the temperature-dependent thermal conductivity, variable reactive index, and heat generation," Heat Transfer-Asian Research, vol. 41, no. 3, pp. 241-259, 2012. 
[24] A. A. Mutlag, M. J. Uddin, M. A. A. Hamad, and A. I. M. Ismail, "Heat transfer analysis for falkner-skan boundary layer flow past a stationary wedge with slips boundary conditions considering temperature-dependent thermal conductivity," Sains Malaysiana, vol. 42, no. 6, pp. 855-862, 2013.

[25] S. C. Cowin, "Polar fluids," Physics of Fluids, vol. 11, no. 9, pp. 1919-1927, 1968.

[26] M. A. Seddeek, M. Y. Akl, and A. M. Al-Hanaya, "Thermal radiation effects on mixed convection and mass transfer flow on vertical porous plate with heat generation and chemical reaction by using scaling group," Journal of Natural Sciences and Mathematics, vol. 4, pp. 41-60, 2010.

[27] M. A. A. Hamad, M. J. Uddin, and A. I. M. Ismail, "Investigation of combined heat and mass transfer by Lie group analysis with variable diffusivity taking into account hydrodynamic slip and thermal convective boundary conditions," International Journal of Heat and Mass Transfer, vol. 55, no. 4, pp. 1355-1362, 2012.

[28] M. J. Uddin, W. A. Khan, and A. I. M. Ismail, "MHD free convective boundary layer flow of a nanofluid past a flat vertical plate with Newtonian heating boundary condition," PLoS ONE, vol. 7, no. 11, Article ID e49499, 2012.

[29] S. Mukhopadhyay and G. C. Layek, "Effects of variable fluid viscosity on flow past a heated stretching sheet embedded in a porous medium in presence of heat source/sink," Meccanica, vol. 47, no. 4, pp. 863-876, 2012.

[30] M. J. Uddin, W. A. Khan, and A. I. M. Ismail, "Scaling group transformation for MHD boundary layer slip flow of a nanofluid over a convectively heated stretching sheet with heat generation," Mathematical Problems in Engineering, vol. 2012, Article ID 934964, 20 pages, 2012.

[31] M. M. Rashidi, M. Ferdows, A. B. Parsa, and S. Abelman, "MHD natural convection with convective surface boundary condition over a at plate," Abstract and Applied Analysis, vol. 2014, Article ID 923487, 10 pages, 2014.

[32] R. E. Bellman and R. E. Kalaba, Quasilinearisation and NonLinear Boundary-Value Problems, Elsevier, New York, NY, USA, 1965.

[33] S. S. Motsa, P. G. Dlamini, and M. Khumalo, "Spectral relaxation method and spectral quasilinearization method for solving unsteady boundary layer flow problems," Advances in Mathematical Physics, vol. 2014, Article ID 341964, 12 pages, 2014.

[34] S. S. Motsa, P. Sibanda, J. M. Ngnotchouye, and G. T. Marewo, "A spectral relaxation approach for unsteady boundary-layer flow and heat transfer of a nanofluid over a permeable stretching/shrinking sheet," Advances in Mathematical Physics, vol. 2014, Article ID 564942, 10 pages, 2014.

[35] C. Canuto, M. Y. Hussaini, A. Quarteroni, and T. Zang, Spectral Methods. Fundamentals in Single Domains, Scientific Computation, Springer, Berlin, Germany, 2006.

[36] J. H. Merkin, "Free convection boundary layer on an isothermal horizontal cylinder," in Proceedings of the American Society of Mechanical Engineers and American Institute of Chemical Engineers, Heat Transfer Conference, p. 911, ASME, St. Louis, Mo, USA, August 1976.

[37] R. Nazar, N. Amin, and I. Pop, "Free convection boundary layer on an isothermal horizontal circular cylinder in a micropolar fluid," in Proceedings of the 12th International Heat Transfer Conference, vol. 2, pp. 525-530, Elsevier, Paris, France, August 2002.

[38] M. M. Molla, M. A. Hossain, and M. C. Paul, "Natural convection flow from an isothermal horizontal circular cylinder in presence of heat generation," International Journal of Engineering Science, vol. 44, no. 13-14, pp. 949-958, 2006. 

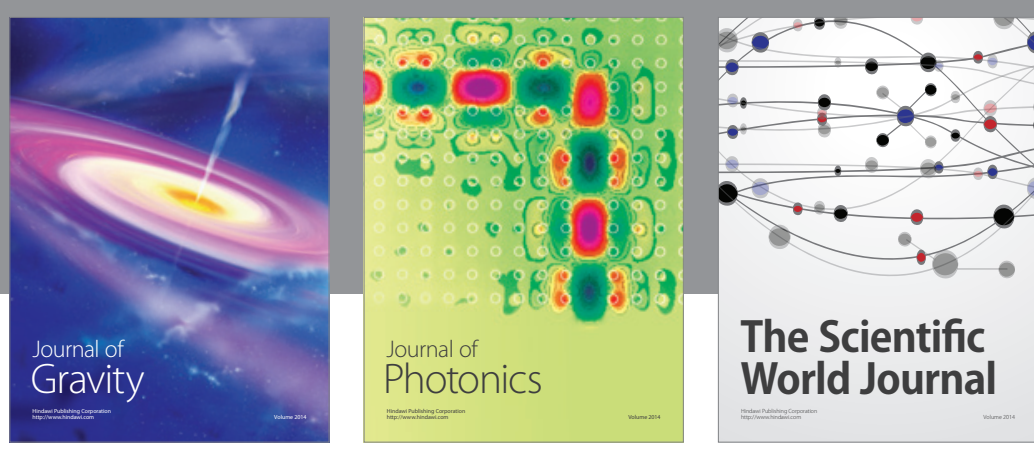

The Scientific World Journal
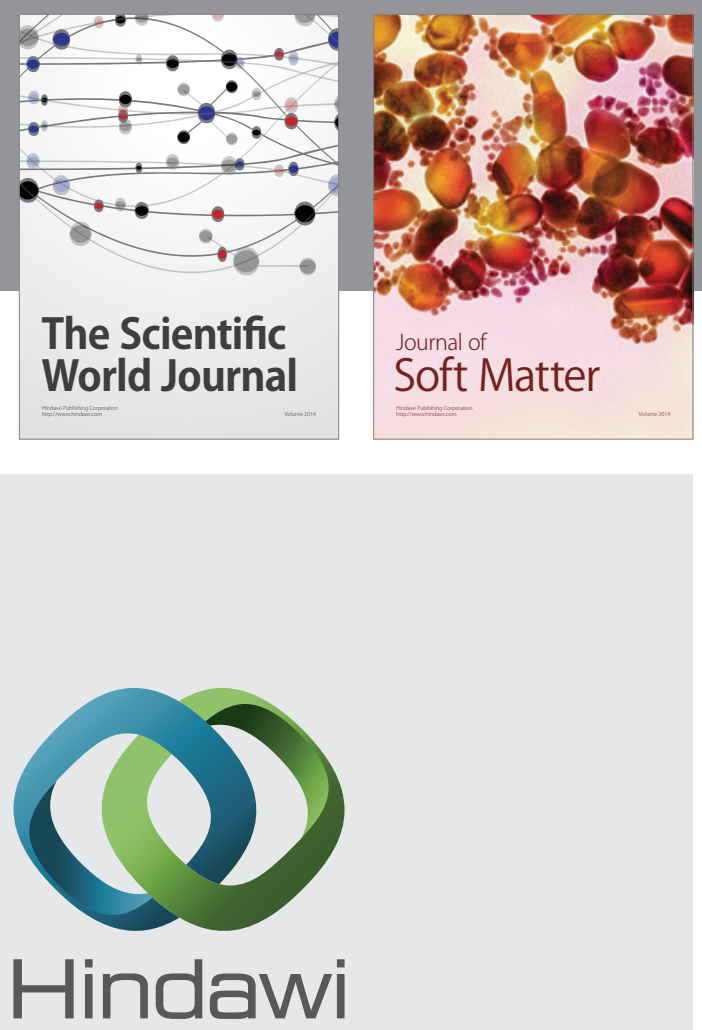

Submit your manuscripts at

http://www.hindawi.com

nternational Journal of

Statistical Mechanics
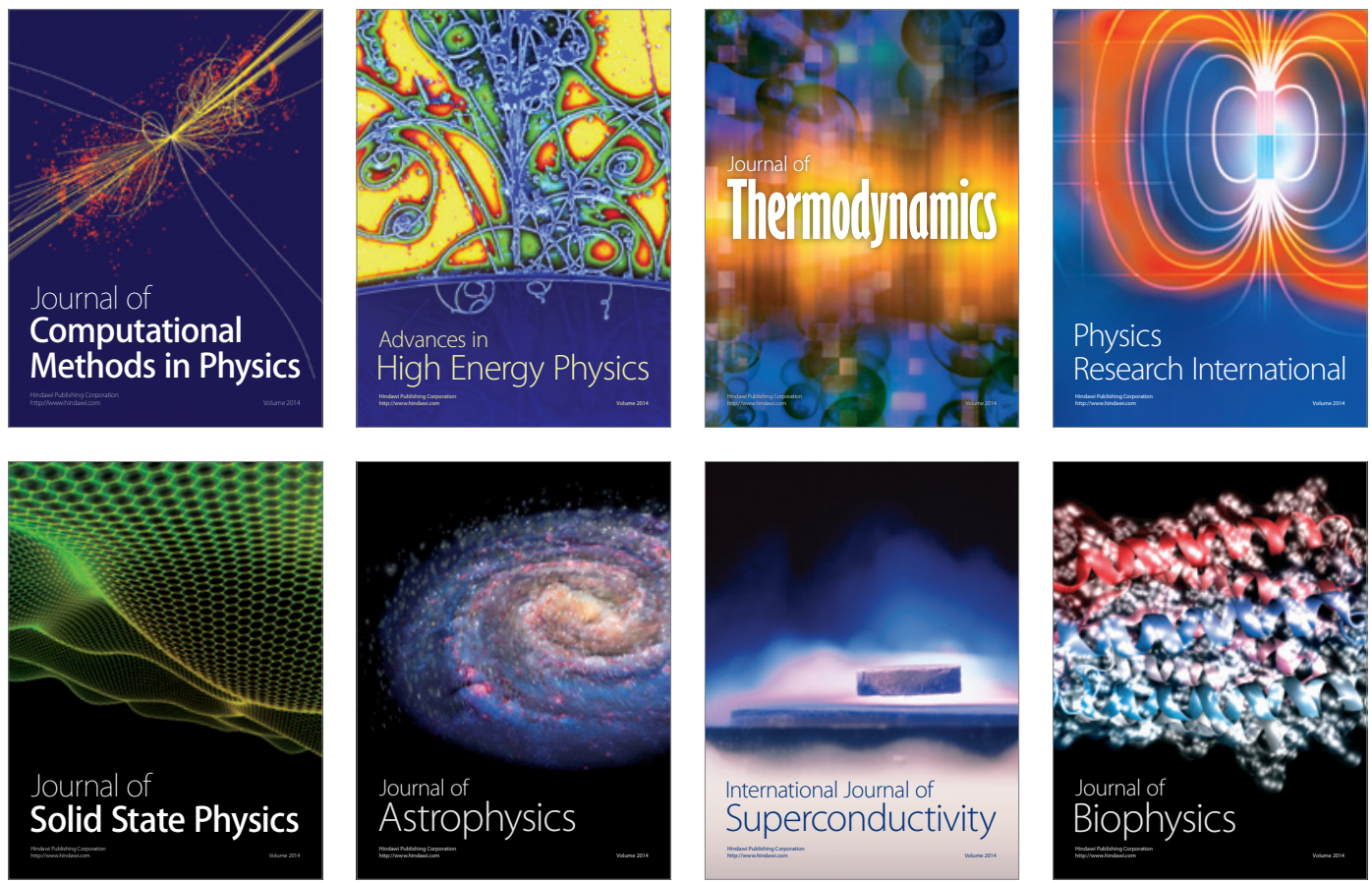
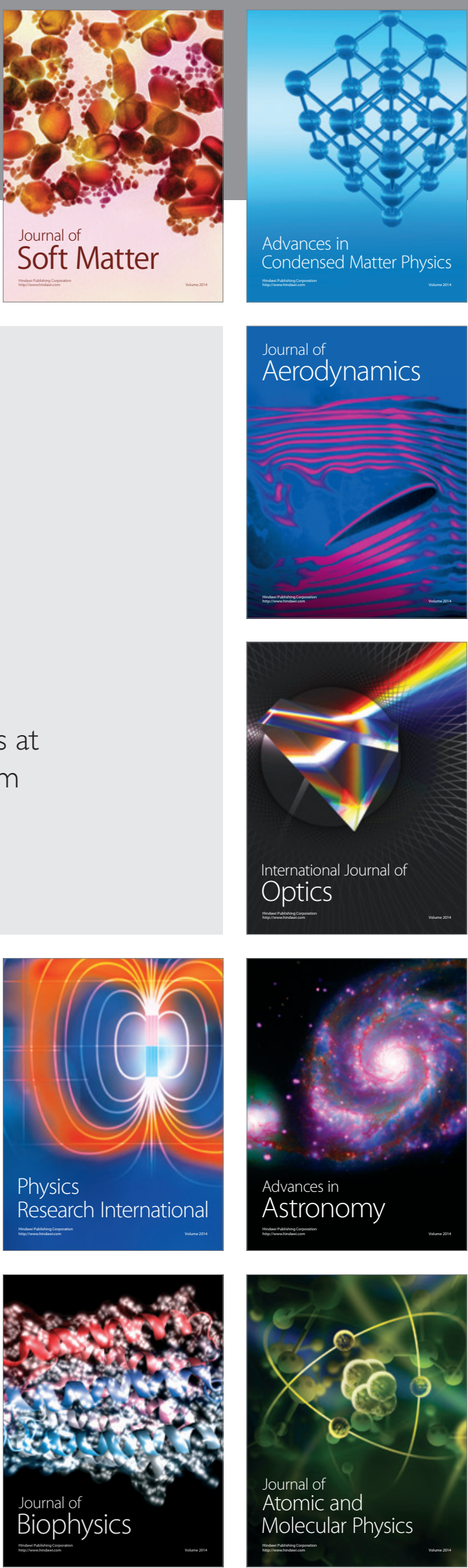